\title{
CONSISTENCY OF THE MAXIMUM LIKELIHOOD ESTIMATE FOR NON-HOMOGENEOUS MARKOV-SWITCHING MODELS
}

\author{
Pierre Ailliot $^{1}$ And Françoise PÈne ${ }^{2}$
}

\begin{abstract}
We prove the consistency of the maximum likelihood estimator for a large family of models generalizing the well known Markov-switching AutoRegressive (MS-AR) models by letting the transition probabilities vary in time and depend on covariates. We illustrate our theoretical result on the famous MacKenzie River lynx dataset and on a multi-site model for downscaling rainfall.
\end{abstract}

Mathematics Subject Classification. 62F12, 62M05.

Received January 15, 2014. Revised September 12, 2014.

\section{INTRODUCTION}

Many nonlinear time series models have been proposed in the last decades. Among them, the models with regime switchings provide a class of versatile and interpretable models which have received an extensive interest in the literature. One of the most influential papers in this field is the one by Hamilton in 1989 (see [13]) where Markov-Switching AutoRegressive (MS-AR) models were introduced. It became one of the most popular nonlinear time series model. MS-AR models combine several autoregressive models to describe the evolution of the observed process $\left\{Y_{k}\right\}$ at different periods of time, the transition between these autoregressive models being controlled by a hidden Markov chain $\left\{X_{k}\right\}$. In most applications, it is assumed that $\left\{X_{k}\right\}$ is an homogeneous Markov chain. In this work, we relax this assumption and let the evolution of $\left\{X_{k}\right\}$ depend on lagged values of $\left\{Y_{k}\right\}$ and exogenous covariates. A particular impetus for this study was the modeling of wind time series (see [3]). These time series are subject to switchings related to meteorological regimes (or "weather types") and it is natural to let these switchings depend on covariates such as the wind direction.

More formally, we assume that $X_{k}$ takes its values in a compact metric space $E$ endowed with a finite Borel measure $\mathfrak{m}_{E}$ and that $Y_{k}$ takes its values in a complete separable metric space $K$ endowed with a non-negative Borel $\sigma$-finite measure $\mathfrak{m}_{K}$ and we set $\mu_{0}:=\mathfrak{m}_{E} \times \mathfrak{m}_{K}$. It will be useful to denote $Y_{k}^{k+\ell}:=\left(Y_{k}, \ldots, Y_{k+\ell}\right), y_{k}^{k+\ell}:=$ $\left(y_{k}, \ldots, y_{k+\ell}\right.$ ) (and to use analogous notations $X_{k}^{k+\ell}, x_{k}^{k+\ell}$ ) for integer $k$ and $\ell \geq 0$. The Non-Homogeneous Markov-Switching AutoRegressive (NHMS-AR) model of order $s>0$ considered in this work is characterized by Hypothesis 1 below.

Hypothesis 1. The sequence $\left\{X_{k}, Y_{k}\right\}_{k}$ is a Markov process of order $s$ with values in $E \times K$ such that, for some parameter $\theta$ belonging to some subset $\Theta$ of $\mathbb{R}^{d}$,

Keywords and phrases. Markov-switching autoregressive process, non-homogeneous hidden Markov process, maximum likelihood, consistency, stability, lynx data.

1 Université de Brest, UMR 6205, 29019 Brest, France. pierre.ailliot@univ-brest.fr

2 Université de Brest and IUF, UMR 6205, 29019 Brest, France. francoise.pene@univ-brest.fr 
- the conditional distribution of $X_{k}$ (wrt $\mathfrak{m}_{E}$ ) given the values of $\left\{X_{k^{\prime}}=x_{k^{\prime}}\right\}_{k^{\prime}<k}$ and $\left\{Y_{k^{\prime}}=y_{k^{\prime}}\right\}_{k^{\prime}<k}$ only depends on $x_{k-1}$ and $y_{k-s}^{k-1}$ and this conditional distribution has a probability density function (pdf) denoted $p_{1, \theta}\left(x_{k} \mid x_{k-1}, y_{k-s}^{k-1}\right)$ with respect to $\mathfrak{m}_{E}$.

- the conditional distribution of $Y_{k}$ given the values of $\left\{Y_{k^{\prime}}=y_{k^{\prime}}\right\}_{k^{\prime}<k}$ and $\left\{X_{k^{\prime}}=x_{k^{\prime}}\right\}_{k^{\prime} \leq k}$ only depends on $x_{k}$ and $y_{k-s}^{k-1}$ and this conditional distribution has a pdf $p_{2, \theta}\left(y_{k} \mid x_{k}, y_{k-s}^{k-1}\right)$ with respect to $\mathfrak{m}_{K}$.

Let us write $q_{\theta}\left(\cdot \mid x_{k-1}, y_{k-s}^{k-1}\right)$ for the conditional pdf (with respect to $\left.\mu_{0}\right)$ of $\left(X_{k}, Y_{k}\right)$ given $\left(X_{k-1}=\right.$ $\left.x_{k-1}, Y_{k-s}^{k-1}=y_{k-s}^{k-1}\right)$. Hypothesis 1 implies that

$$
q_{\theta}\left(x, y \mid x_{k-1}, y_{k-s}^{k-1}\right)=p_{1, \theta}\left(x \mid x_{k-1}, y_{k-s}^{k-1}\right) p_{2, \theta}\left(y \mid x, y_{k-s}^{k-1}\right) .
$$

The various conditional independence assumptions of Hypothesis 1 are summarized by the directed acyclic graph (DAG) below when $s=1$.

$\begin{array}{lll}\text { Hidden Regime } & \cdots \rightarrow X_{k-1} \rightarrow X_{k} \rightarrow X_{k+1} \rightarrow \cdots \\ \text { Observed time series } & \cdots & \downarrow Y_{k-1} \rightarrow Y_{k} \rightarrow Y_{k+1} \rightarrow \cdots\end{array}$

This defines a general family of models which encompasses the most usual models with regime switchings.

- When $p_{1, \theta}\left(x_{k} \mid x_{k-1}, y_{k-s}^{k-1}\right)$ does not depend on $y_{k-s}^{k-1}$, the evolution of the hidden Markov chain $\left\{X_{k}\right\}$ is homogeneous and independent of the observed process and we retrieve the usual MS-AR models. If we further assume that $p_{2, \theta}\left(y_{k} \mid x_{k}, y_{k-s}^{k-1}\right)$ does not depend of $y_{k-s}^{k-1}$, we obtain the Hidden Markov Models (HMMs).

- When $p_{1, \theta}\left(x_{k} \mid x_{k-1}, y_{k-s}^{k-1}\right)$ does not depend on $x_{k-1}$ and is parametrized using indicator functions, we obtain the Threshold AutoRegressive (TAR) models which is another important family of models with regime switching in the literature (see e.g. [23]).

HMMs, MS-AR and TAR models have been used in many fields of applications and their theoretical properties have been extensively studied (see e.g. $[6,11,23]$ ).

Models with non-homogeneous Markov switchings have also been considered in the literature. In particular, they have been used to describe breaks associated with events such as financial crises or abrupt changes in government policy in econometric time series (see [15] and references therein). In these models, generally referred to as models with time varying transitions probabilities, the switching probabilities depend on variables which describe the strength of the economy or leading indicators of change. They are also popular for meteorological applications with the regimes describing the so-called "weather types". The switchings generally depend on largescale variables (see e.g. $[2,5,14,25])$ for downscaling applications but may also depend on the past observations (see [3]). The most usual method procedure to fit such models consists in computing the Maximum Likelihood Estimates (MLE). It is indeed relatively straightforward to adapt the standard numerical estimation techniques which are available for the homogeneous models, such as the forward-backward recursions or the EM algorithm, to the non-homogeneous models (see e.g. $[8,14,15]$ ). However, we could not find any theoretical results on the asymptotic properties of the MLE for these models and this paper aims at filling this gap.

The paper is organized as follows. In Section 2, we give general conditions ensuring the consistency of the MLE. In Sections 3 and 4, we show that these general conditions apply to various specific but representative NHMS-AR models. Some results are proven in the appendices.

\section{A general CONSistency RESUlt of MLE for NHMS-AR MOdels}

We aim at estimating the true parameter $\theta^{*} \in \Theta$ of a NHMS-AR process $\left(X_{k}, Y_{k}\right)_{k}$ for which only the component $\left\{Y_{k}\right\}$ is observed. For that we consider the Maximum Likelihood Estimator (MLE) $\hat{\theta}_{n, x_{0}}$ which is 
defined as the maximizer of $\theta \mapsto \ell_{n}\left(\theta, x_{0}\right)$ for a fixed $x_{0} \in E$ with

$$
\ell_{n}\left(\theta, x_{0}\right)=\log p_{\theta}\left(Y_{1}^{n} \mid X_{0}=x_{0}, Y_{-s+1}^{0}\right)=\sum_{k=1}^{n} \log \frac{p_{\theta}\left(Y_{1}^{k} \mid X_{0}=x_{0}, Y_{-s+1}^{0}\right)}{p_{\theta}\left(Y_{1}^{k-1} \mid X_{0}=x_{0}, Y_{-s+1}^{0}\right)},
$$

where $p_{\theta}\left(Y_{1}^{k} \mid X_{0}=x_{0}, Y_{-s+1}^{0}\right)$ is the conditional pdf of $Y_{1}^{k}$ given $\left(X_{0}=x_{0}, Y_{-s+1}^{0}\right)$ evaluated at $Y_{1}^{k}$, i.e.

$$
p_{\theta}\left(Y_{1}^{k} \mid X_{0}=x_{0}, Y_{-s+1}^{0}\right):=\int_{E^{k}} \prod_{\ell=1}^{k} q_{\theta}\left(x_{\ell}, Y_{\ell} \mid x_{\ell-1}, Y_{\ell-s}^{\ell-1}\right) d \mathfrak{m}_{E}^{\otimes k}\left(x_{1}^{k}\right) .
$$

Observe that $\ell_{n}\left(\theta, x_{0}\right)$ is a random variable depending on $Y_{-s+1}^{0}$ (which is observed).

Before stating our main result, we introduce quickly some notations (see beginning of Appendix A for further details). Let $Q_{\theta}$ be the transition operator of the $s$-order Markov process $\left(X_{k}, Y_{k}\right)_{k}, Q_{\theta}$ being seen as an operator acting on the set of complex-valued bounded measurable functions on $E \times K^{s}$ (or on some other complex Banach space) and let $Q_{\theta}^{*}$ be its adjoint operator. We set $\mu:=\mathfrak{m}_{E} \times \mathfrak{m}_{K}^{\otimes s}$. We identify $\left(X_{k}, Y_{k}\right)_{k}$ with the canonical Markov chain. We suppose that, for every $\theta \in \Theta$, there exists a unique stationary probability $\bar{\nu}_{\theta}$ for the Markov chain with transition operator $Q_{\theta}$ (i.e. $\bar{\nu}_{\theta}$ is an invariant probability measure for $Q_{\theta}^{*}$ ) with pdf $h_{\theta}$ with respect to $\mu$. We then write $\overline{\mathbb{P}}_{\theta}$ for the probability measure corresponding to this invariant probability and $\overline{\mathbb{P}}_{\theta}^{Y}$ for the distribution of $Y$ with respect to $\overline{\mathbb{P}}_{\theta}$. For every $(x, y) \in E \times K^{s}$ and any integer $k \geq s$, we write $Q^{* k}(\cdot \mid x, y)$ for the pdf of $\left(X_{k}, Y_{k}^{k+s-1}\right)$ with respect to $\mu$ given $\left(X_{0}, Y_{0}^{s-1}\right)=(x, y)$.

The question of consistency of the MLE has been studied by many authors in the context of usual HMMs (see e.g. $[9,18,19]$ ) and MS-AR models (see [10] and references therein). The aim of this section is to state consistency results of MLE for general NHMS-AR. The proof of the following theorem is a careful adaptation of the proof of ([10], Thms. 1 and 5). This proof is given in Appendix A.

Theorem 2.1. Assume that $\Theta$ is compact, that there exists a unique invariant probability measure for every $\theta \in \Theta$, that $\overline{\mathbb{P}}_{\theta^{*}}$ is absolutely continuous with respect to $\overline{\mathbb{P}}_{\theta}$ for every $\theta \in \Theta$, that $p_{1}$ and $p_{2}$ are continuous in $\theta$. Assume also that the following conditions hold true

$$
\begin{gathered}
0<p_{1,-}:=\inf _{\theta, x_{1}, x_{0}, y_{0}} p_{1, \theta}\left(x_{1} \mid x_{0}, y_{0}\right) \leq p_{1,+}:=\sup _{\theta, x_{1}, x_{0}, y_{0}} p_{1, \theta}\left(x_{1} \mid x_{0}, y_{0}\right)<\infty, \\
B_{-}:=\overline{\mathbb{E}}_{\theta^{*}}\left[\left|\log \left(\inf _{\theta} \int_{E} p_{2, \theta}\left(Y_{0} \mid x_{0}, Y_{-s}^{-1}\right) d \mathfrak{m}_{E}\left(x_{0}\right)\right)\right|\right]<\infty \\
B_{+}:=\overline{\mathbb{E}}_{\theta^{*}}\left[\left|\log \left(\sup _{\theta} \int_{E} p_{2, \theta}\left(Y_{0} \mid x_{0}, Y_{-s}^{-1}\right) d \mathfrak{m}_{E}\left(x_{0}\right)\right)\right|\right]<\infty \\
\forall \theta \in \Theta, \sup _{y_{-s}^{-1}} \int_{E} p_{2, \theta}\left(Y_{0} \mid x, y_{-s}^{-1}\right) d \mathfrak{m}_{E}(x)<\infty, \overline{\mathbb{P}}_{\theta^{*}}-a . s \\
\forall \theta \in \Theta, \text { for } \mu-\text { a.e. }(x, y) \in E \times K^{s}, \lim _{k \rightarrow+\infty} \| Q_{\theta}^{* k}(\cdot \mid x, y)-h_{\theta}||_{L^{1}(\mu)}=0 .
\end{gathered}
$$

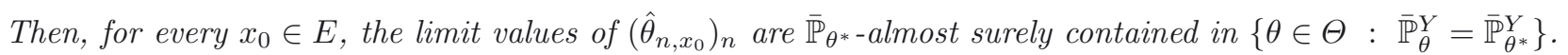

If, moreover, $Q_{\theta^{*}}$ is positive Harris recurrent and aperiodic, then, for every $x_{0} \in E$ and every initial probability $\nu$, the limit values of $\left(\hat{\theta}_{n, x_{0}}\right)_{n}$ are almost surely contained in $\left\{\theta \in \Theta: \overline{\mathbb{P}}_{\theta}^{Y}=\overline{\mathbb{P}}_{\theta^{*}}^{Y}\right\}$.

Our hypotheses are close to those of [10]. Let us point out the main differences. First, in [10] $p_{1, \theta}\left(x \mid x^{\prime}, y^{\prime}\right)$ does not depend on $y^{\prime}$. Second, (2.3) and (2.4) are slightly weaker than

$$
\sup _{\theta, y_{-s}^{-1}, y_{0}, x} p_{2, \theta}\left(y_{0} \mid x, y_{-s}^{-1}\right)<\infty
$$


assumed in [10]. This is illustrated below in Section 4 where the parametrization of $p_{2}$ uses Gamma pdf which may not be bounded close to the origin depending on the values of the parameters. The results given in [10] do not apply directly to this model whereas we will show that (2.3) applies (see also [1]). Third, (2.5) is slightly weaker than the positive Harris recurrence. Let us precise that (2.5) is equivalent to any of the two following properties

- for any initial measure $\nu$ on $E \times K^{s}$, we have $\lim _{n \rightarrow+\infty}\left\|Q_{\theta}^{* n} \nu-\nu_{\theta}\right\|_{T V}=0$, where $\|\cdot\|_{T V}$ stands for the total variation norm,

- for any initial measure $\nu$ on $E \times K^{s}$, we have $\lim _{n \rightarrow+\infty} \sup _{\nu \in \mathcal{P}(E \times K)}\left\|\left[Q_{\theta}^{* n} \nu\right]-h_{\theta}\right\|_{L^{1}\left(\mathfrak{m}_{E} \times \mathfrak{m}_{K}^{s}\right)}=0$, with $\mathcal{P}(E \times K)$ the set of probability measures on $E \times K$.

Remark 2.2. Observe that, if $q_{\theta}>0$ and if $\nu_{\theta}$ exists for every $\theta \in \Theta$, then the pdf $h_{\theta}$ of $\nu_{\theta}$ satisfies $h_{\theta}>0$ $\left(\mu\right.$-a.e.). In this case, $\overline{\mathbb{P}}_{\theta^{*}}$ is absolutely continuous with respect to $\overline{\mathbb{P}}_{\theta}$ for every $\theta \in \Theta$.

In our applications, the set $E$ will be finite and $\mathfrak{m}_{E}$ will be the counting measure. In this case (2.1)-(2.4) can be rewritten as follows

$$
\begin{array}{r}
\forall x_{1}, x_{0} \in E, \quad 0<\inf _{\theta, y_{0}} p_{1, \theta}\left(x_{1} \mid x_{0}, y_{0}\right) \leq \sup _{\theta, y_{0}} p_{1, \theta}\left(x_{1} \mid x_{0}, y_{0}\right)<\infty, \\
\forall x \in E, \quad \overline{\mathbb{E}}_{\theta^{*}}\left[\left|\log \left(\inf _{\theta} p_{2, \theta}\left(Y_{0} \mid x, Y_{-s}^{-1}\right)\right)\right|\right]+\overline{\mathbb{E}}_{\theta^{*}}\left[\left|\log \left(\sup _{\theta} p_{2, \theta}\left(Y_{0} \mid x, Y_{-s}^{-1}\right)\right)\right|\right]<\infty, \\
\forall x \in E, \forall \theta \in \Theta, \sup _{\substack{-1 \\
y_{-s}}}^{-1}\left(Y_{0} \mid x, y_{-s}^{-1}\right)<\infty, \overline{\mathbb{P}}_{\theta^{*}}-\text { a.s. }
\end{array}
$$

\section{NHMS-AR MODEL WITH LINEAR AUTOREGRESSIVE MODELS}

\subsection{A NHMS-AR model for MacKenzie River lynx data}

In this section we introduce a particular NHMS-AR model and discuss the results obtained when fitting this model to the the time series of annual number of Canadian lynx trapped in the Mackenzie River district of northwest Canada from 1821 to 1934. This time series is a benchmark dataset to test nonlinear time series model (see e.g. $[11,23])$. In order to facilitate the comparison with the other works on this time series, we analyze the data at the logarithmic scale with the base 10 shown in Figure 1. This time series exhibits periodic fluctuations (it may be due to the competition between several species, predator-prey interaction,... ) with asymmetric cycles (increasing phase are slower than decreasing phase) which makes it challenging to model.

In [23], it was proposed to fit a $\operatorname{SETAR}(2)$ model to this time series. The fitted model is the following

$$
Y_{k}=\left\{\begin{array}{l}
0.51+1.23 Y_{k-1}-0.37 Y_{k-2}+0.18 \epsilon_{k}\left(Y_{k-2} \leq 3.15\right) \\
2.32+1.53 Y_{k-1}-1.27 Y_{k-2}+0.23 \epsilon_{k}\left(Y_{k-2}>3.15\right)
\end{array}\right.
$$

The two regimes have a nice biological interpretation in terms of prey-predator interaction, with the upper regime $\left(Y_{t-2}>3.15\right)$ corresponding to a population decrease whereas the population tends to increase in the lower regime.

The NHMS-AR model defined below has been fitted to this time series.

Hypothesis 2. We assume that $E=\{1,2\}$ (endowed with the counting measure), $K=\mathbb{R}$ (endowed with the Lebesgue measure) and $\left\{Y_{k}\right\}$ satisfies

$$
Y_{k}=\beta_{0}^{\left(x_{k}\right)}+\sum_{\ell=1}^{s} \beta_{\ell}^{\left(x_{k}\right)} Y_{k-\ell}+\sigma^{\left(x_{k}\right)} \epsilon_{k},
$$



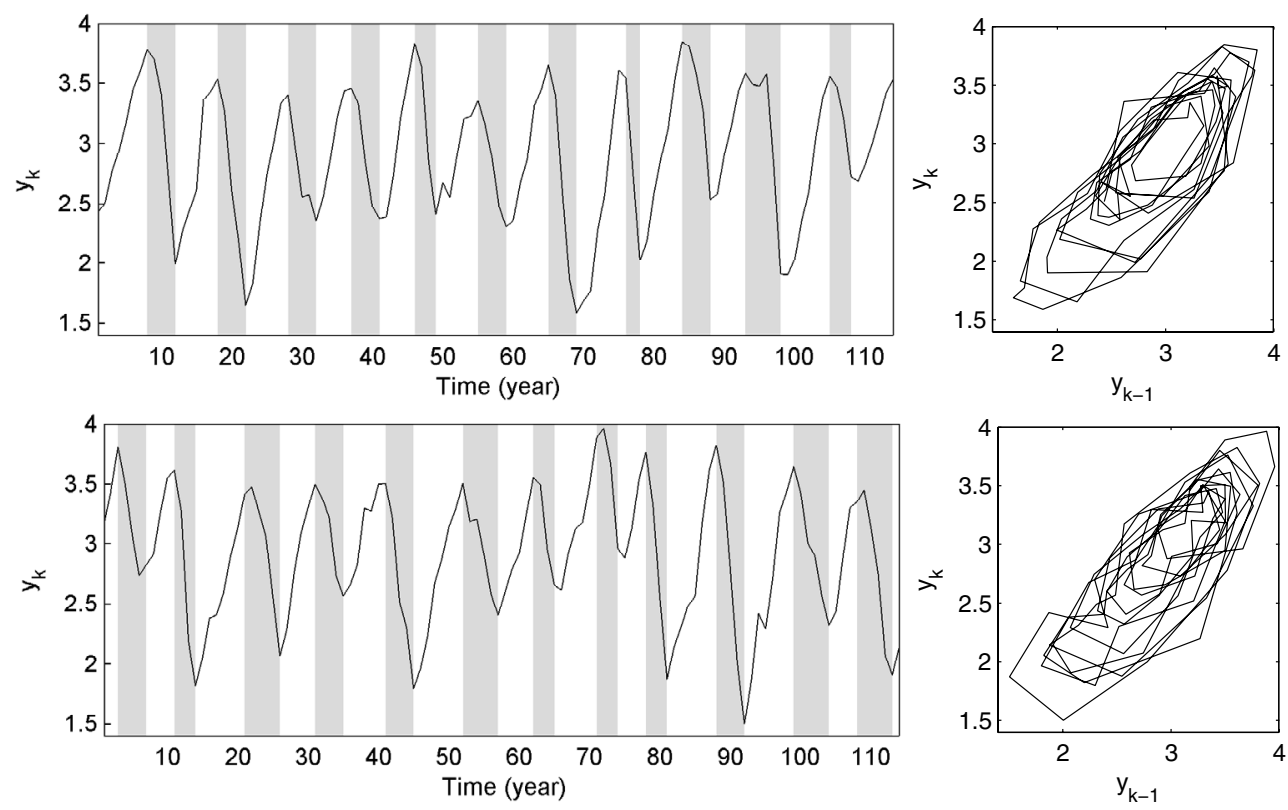

Figure 1. Top left panel: time plot of log Canadian lynx data. The color indicates the most likely regimes identified by the fitted NHMS-AR model. The first (resp. second) regime is the most likely when the color is white (resp. gray). Top right panel: directed scatter plot of log Canadian lynx data. Bottom left panel: time plot of a sequence simulated with the fitted NHMS-AR model data. The color indicates the simulated regime (first regime in white, second regime in gray). Bottom right panel: directed scatter plot of the simulated sequence shown on the bottom left panel.

with $\left\{\epsilon_{k}\right\}$ an iid sequence of standard Gaussian random variables, with $\sigma^{(x)}>0$ and $\beta_{l}^{(x)} \in \mathbb{R}$ for every $\ell \in\{0, \ldots, s\}$ and every $x \in\{1,2\}$,

$$
\text { i.e. } \quad p_{2, \theta}\left(y_{k} \mid x_{k}, y_{k-s}^{k-1}\right)=\mathcal{N}\left(y_{k} ; \beta_{0}^{\left(x_{k}\right)}+\sum_{\ell=1}^{s} \beta_{\ell}^{\left(x_{k}\right)} y_{k-\ell}, \sigma^{\left(x_{k}\right)}\right),
$$

where $\mathcal{N}(\cdot ; \beta, \sigma)$ stands for the Gaussian pdf with mean $\beta$ and standard deviation $\sigma$.

The transition probabilities of $\left\{X_{k}\right\}$ are parametrized using the logistic function as follows when $x_{k}=x_{k-1}$

$$
p_{1, \theta}\left(x_{k} \mid x_{k-1}, y_{k-s}^{k-1}\right)=\pi_{-}^{\left(x_{k-1}\right)}+\frac{1-\pi_{-}^{\left(x_{k-1}\right)}-\pi_{+}^{\left(x_{k-1}\right)}}{1+\exp \left(\lambda_{0}^{\left(x_{k-1}\right)}+\lambda_{1}^{\left(x_{k-1}\right)} y_{k-r}\right)},
$$

with $r \leq s$ a positive integer and the unknown parameters $\pi_{-}^{(x)}, \pi_{+}^{(x)}, \lambda_{0}^{(x)}, \lambda_{1}^{(x)}$ for $x \in\{1,2\}$.

The unknown parameter $\theta$ corresponds to

$$
\theta=\left(\left(\beta_{i}^{(x)}\right),\left(\sigma^{(x)}\right),\left(\pi_{-}^{(x)}\right),\left(\pi_{+}^{(x)}\right),\left(\lambda_{i}^{(x)}\right)\right) .
$$

We write $\tilde{\Theta}$ for the set of such parameters $\theta$ satisfying, for every $x \in\{1,2\}, \sigma^{(x)}>0$ and $0<\pi_{-}^{(x)}<1-\pi_{+}^{(x)}<1$ (this last constraint is added in order to ensure that (2.1) holds). 
Although very simple, this model encompasses the homogeneous Gaussian MS-AR model when $\lambda_{1}^{(1)}=\lambda_{1}^{(2)}=0$ and the $\operatorname{SETAR}(2)$ model as a limit case. Indeed, if $s_{0}=-\frac{\lambda_{0}^{(x)}}{\lambda_{1}^{(x)}}$ is fixed for $x \in\{1,2\}, \lambda_{1}^{(1)} \rightarrow+\infty, \lambda_{1}^{(2)} \rightarrow-\infty$, $\pi_{-}^{(x)} \rightarrow 0$ and $\pi_{+}^{(x)} \rightarrow 0$ then

$$
p_{1}\left(X_{k}=1 \mid x_{k-1}, y_{k-s}^{k-1}\right) \rightarrow \mathbb{1}\left(y_{k-r} \leq s_{0}\right) \text { and } p_{1}\left(X_{k}=2 \mid x_{k-1}, y_{k-s}^{k-1}\right) \rightarrow \mathbb{1}\left(y_{k-r} \geq s_{0}\right) .
$$

Both models have been extensively studied in the literature.

In practice, we have used the EM algorithm to compute the MLE. The recursions of this algorithm are relatively similar to the ones of the MS-AR model (see $[8,17]$ ). To facilitate the comparison with the SETAR $(2)$ model (3.1), we have also considered AR models of order $s=2$ and a lag $r=2$ for the transition probabilities. The fitted model is the following

$$
Y_{k}=\left\{\begin{array}{llccc}
0.54 & +1.11 & Y_{k-1}-0.24 & Y_{k-2}+0.14 & \epsilon_{k}\left(X_{k}=1\right) \\
(0.31,0.80) & (0.96,1.27) & (-0.43,-0.05) & (0.11,0.17) & \\
1.03 & +1.49 & Y_{k-1}-0.87 & Y_{k-2}+0.22 & \epsilon_{k}\left(X_{k}=2\right) \\
(-0.12,1.86) & (1.23,1.69) & (-1.20,-0.39) & (0.14,0.26) &
\end{array}\right.
$$

with

$$
P\left(X_{k}=i \mid X_{k-1}=i, Y_{k-2}=y_{k-2}\right)=\left\{\begin{array}{ccc}
(1+\exp (-42.4+12.8 & \left.\left.y_{k-2}\right)\right)^{-1} \\
& (-587,-16.3) & (4.77,176) \\
(1+\exp (9.07 & -3.33 & \left.\left.y_{k-2}\right)\right)^{-1} \\
& (2.25,178) & (-64.1,-1.12),
\end{array} \quad\left(X_{k}=2\right)\right.
$$

where the italic values in parenthesis below the parameter values correspond to $95 \%$ confidence intervals computed using parametric bootstrap (see e.g. [24]). These values reflect the finite sample properties of the estimates. The estimate of $\pi_{-}^{(x)}$ and $\pi_{+}^{(x)}$ are not given because they are very close to 0 . It means that these technical parameters have no practical importance and can be fixed equal to an arbitrary small value (here we used the machine epsilon $2^{-52}$ ). There are small differences between the AR coefficients (3.1) and (3.4) but the dynamics inside the regimes of the SETAR(2) and NHMS-AR models are broadly similar. The models differ mainly in the mechanism used to govern the switchings between the two regimes. For the SETAR model the regime is a deterministic function of a lagged value of the observed process. The NHMS-AR model can be seen as a fuzzy extension of the SETAR model where the regime has its own Markovian evolution influenced by the lagged value of the observed process. This is illustrated in Figure 2 which shows the transition probabilities (3.5) and the threshold of the SETAR(2) model. According to this figure, it seems reasonable to model the transition from regime 1 to regime 2 by a step function at the level $y_{k-2} \approx 3.15$ but the values of $y_{k-2}$ for which the transition from regime 2 to regime 1 occurs seem to be more variable and the step function approximation less realistic.

The asymmetries in the cycle imply that the system spends less time in the second regime (decreasing phase) than in the first one. It may explain the larger confidence intervals in the second regime compared to the first one (see (3.4)). Figure 2 shows that there is an important sampling variability in the estimate of the transition kernel of the hidden process. This is probably due to the low number of transitions among regimes (see Fig. 1) which makes it difficult to estimate the associated parameters. A similar behavior has been observed when fitting the model to other time series.

Table 1 gives the AIC and BIC values defined as

$$
A I C=-2 \log L+2 d, \quad B I C=-2 \log L+d \log (N),
$$

where $L$ is the likelihood of the data and $N$ is the number of observations, recall that $d$ is the number of parameters. The values for the NHMS-AR and SETAR models are relatively similar. The NHMS-AR models 

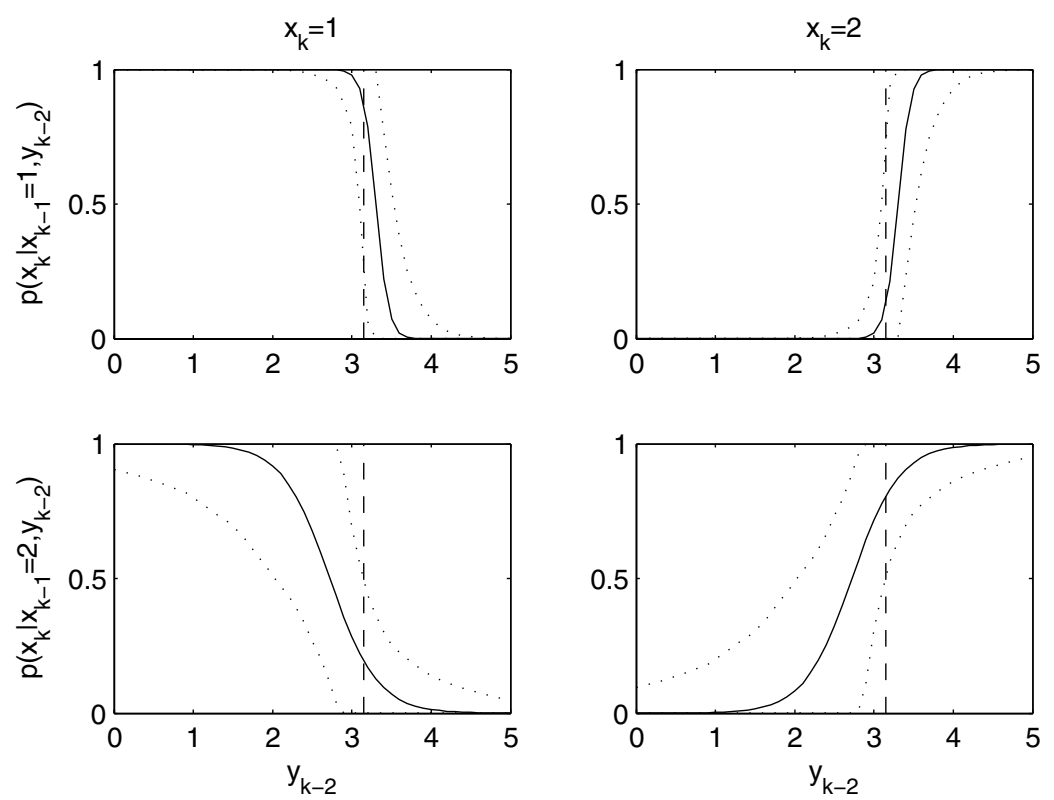

FIgURE 2. Transition probabilities $P\left(X_{k}=j \mid X_{k-1}=i, Y_{k-2}=y_{k-2}\right)$ as a function of $y_{k-2}$. The dotted lines correspond to $95 \%$ confidence intervals computed using parametric bootstrap. The dashed vertical line corresponds to the threshold (3.15) of the $\operatorname{SETAR}(2)$ model.

TABLE 1. AIC and BIC values for the fitted SETAR, homogeneous MS-AR and NHMS-AR models.

\begin{tabular}{cccc}
\hline & AIC & BIC & d \\
\hline SETAR $(s=2)$ & -28.33 & -3.70 & 9 \\
MS-AR $(s=2)$ & -0.2063 & 27.15 & 10 \\
NHMS-AR $(r=s=2)$ & -30.83 & 2.00 & 12 \\
\hline
\end{tabular}

has a slightly better AIC value but BIC selects the SETAR model. As expected, these two models clearly outperform the homogeneous MS-AR which does not include information on the past values in the switching mechanism.

The simulated sequence shown in Figure 1 exhibits a similar cyclical behavior than the data. A more systematic validation was performed but the results are hard to analyze because of the low amount of data available. The model can be generalized in several ways to handle $M \geq 3$ regimes or include covariates, for example through a linear function in the logistic term (see e.g. [8]). Other link functions, such as the probit model used in [15] or a Gaussian kernel (see (4.1)), or non-linear autoregressive models could also be considered. Such models have been developed for various environmental data including temperature and wind time series. The fitted models generally provide an accurate description of the distributional properties of these time series and accurate short-term forecasts. This will be the subject of a forthcoming paper.

\subsection{Properties of the Markov chain}

In this section, we discuss the recurrent and ergodic properties of the model introduced in the previous section. It is a key step to prove the consistence of the MLE (see Thm. 2.1). Various authors have studied the ergodicity of MS-AR $[12,26,27]$ and TAR $[4,7]$ models. A classical approach to prove the ergodicity of a non-linear time series consists in establishing a drift condition. Here we will use a strict drift condition. Let $\|\cdot\|$ 
be some norm on $\mathbb{R}^{s}$. For any $R>0$, we consider the set $E_{R}:=\left\{\left(x, y_{-s+1}^{0}\right):\left\|y_{-s+1}^{0}\right\| \leq R\right\}$. Recall that $\mu$ is here the product of the counting measure on $E$ and of the Lebesgue measure on $\mathbb{R}^{s}$.

Proposition 3.1. Under Hypothesis 2, the Markov chain is $\psi$-irreducible (with $\psi=\mu$ ) and for any $R>0$, the set $E_{R}$ is $\nu_{s}$-small and $\nu_{s+1}$-small with $\nu_{s}$ and $\nu_{s+1}$ equivalent to $\mu$. In particular, the Markov chain is aperiodic.

Proof. The $\psi$-irreducibility comes from the positivity of $q_{\theta}$. Let us prove that $E_{R}$ is $\nu_{s}$-small with $\nu_{s}=h_{s} \cdot \mu$ and

$$
h_{s}\left(x_{s}, y_{1}^{s}\right)=\inf _{\left(x_{0}, y_{-s+1}^{0}\right) \in E_{R}} \int_{E^{s}} \prod_{\ell=1}^{s} q_{\theta}\left(x_{\ell}, y_{\ell} \mid x_{\ell-1}, y_{\ell-s}^{\ell-1}\right) d x_{1}^{s-1}>0 .
$$

Indeed $p_{1, \theta}$ is uniformly bounded from below by some $p_{1,-}, \sigma^{(x)}$ are uniformly bounded from above by some $\sigma_{+}$and from below by some $\sigma_{-}$and, for every $\ell \in\{1, \ldots, s\}$, we have

$$
\forall Z \in \mathbb{R}, \quad g_{\ell}(Z):=\sup _{\left(x_{\ell}, y_{-s+1}^{0}\right) \in E_{R}}\left|Z-\beta_{0}^{\left(x_{\ell}\right)}-\sum_{j=\ell}^{s} \beta_{j}^{\left(x_{\ell}\right)} y_{\ell-j}\right|^{2}<\infty .
$$

So

$$
h_{s}\left(x_{s}, y_{1}^{s}\right) \geq \inf _{x_{1}, \ldots, x_{s} \in\{1,2\}} \frac{\left(p_{1,-}\right)^{s}}{\left(2 \pi \sigma_{-}\right)^{\frac{s}{2}}} \exp \left(-\frac{1}{2 \sigma_{+}} \sum_{\ell=1}^{s} g_{\ell}\left(y_{\ell}-\sum_{j=1}^{\ell-1} \beta_{j}^{\left(x_{\ell}\right)} y_{\ell-j}\right)\right) .
$$

The proof of the $\nu_{s+1}$-smallness of $E_{R}$ (with $\nu_{s+1}$ equivalent to $\mu$ ) uses the same ideas.

Now, to obtain the other properties related to the ergodicity of the process for practical applications (including the practical example given in Sect. 3.1), we can use the following strict drift property.

Hypothesis 3. There exist three real numbers $K<1, L>0$ and $R>0$ such that, for every $\left(x_{0}, y_{-s+1}^{0}\right) \in$ $\{1,2\} \times \mathbb{R}^{s}$,

$$
\mathbb{E}\left[\left\|Y_{-s+2}^{1}\right\|^{2} \mid Y_{-s+1}^{0}=y_{-s+1}^{0}, X_{0}=x_{0}\right] \leq K\left\|y_{-s+1}^{0}\right\|^{2}+L \mathbb{1}_{E_{R}}\left(y_{-s+1}^{0}\right) .
$$

Recall that this property has several classical consequences (see [21], Chaps. 11 and 15 for more details). Hypothesis 3 (combined with the irreducibility and aperiodicity coming from Hypothesis 2) implies in particular

- the existence of a (unique) stationary measure admitting a moment of order 2;

- the $V$-geometric ergodicity with $V\left(x, y_{-s+1}^{0}\right)=\left\|y_{-s+1}^{0}\right\|^{2}$;

- the positive Harris recurrence.

We end this section with some comments on (3.6). Let us write

$$
\Lambda^{(x)}=\left(\begin{array}{cccccc}
0 & 1 & 0 & 0 & \cdots & 0 \\
0 & 0 & 1 & 0 & \cdots & 0 \\
\vdots & \vdots & \vdots & \ddots & \vdots & \\
0 & 0 & 0 & 0 & \cdots & 1 \\
\beta_{s}^{(x)} & \beta_{s-1}^{(x)} & \cdots & \cdots & \cdots & \beta_{1}^{(x)}
\end{array}\right)
$$


for the companion matrix associated to the AR model in regime $x$,

$$
\Phi^{(x)}:=\left(\begin{array}{c}
0 \\
0 \\
\vdots \\
0 \\
\beta_{0}^{(x)}
\end{array}\right), \quad \Sigma^{(x)}=\left(\begin{array}{cccc}
0 & \cdots & 0 & 0 \\
\vdots & \vdots & \vdots & \vdots \\
0 & \cdots & 0 & 0 \\
0 & \cdots & 0 & \sigma^{(x)}
\end{array}\right) \text { and } \varepsilon:=\left(\begin{array}{c}
0 \\
0 \\
\vdots \\
0 \\
\varepsilon_{1}
\end{array}\right) \text {. }
$$

There exist $A, B>0$ such that, for every $\left(x_{0}, y_{-s+1}^{0}\right) \in\{1,2\} \times \mathbb{R}^{s}$, we have

$$
\begin{aligned}
\mathbb{E}\left[\left\|Y_{-s+2}^{1}\right\|^{2} \mid Y_{-s+1}^{0}=y_{-s+1}^{0}, X_{0}=x_{0}\right] & =\sum_{x_{1}=1}^{M} p_{1, \theta}\left(x_{1} \mid x_{0}, y_{-s+1}^{0}\right) \mathbb{E}\left[\left\|\Lambda^{\left(x_{1}\right)} y_{-s+1}^{0}+\Phi^{\left(x_{1}\right)}+\Sigma^{\left(x_{1}\right)} \varepsilon\right\|^{2}\right] \\
& \leq \sum_{x_{1}=1}^{M} p_{1, \theta}\left(x_{1} \mid x_{0}, y_{-s+1}^{0}\right)\left\|\Lambda^{\left(x_{1}\right)}\right\|^{2}\left\|y_{-s+1}^{0}\right\|^{2}+A\left\|y_{-s+1}^{0}\right\|+B
\end{aligned}
$$

where $\|$.$\| denotes abusively the matrix norm associated to the vector norm. We deduce the following.$

Remark 3.2. The strict drift condition (3.6) is satisfied when there exists $M>0$ such that for all $x_{0} \in E$ and all $y_{-s+1}^{0} \in \mathbb{R}^{s}$

$$
\left\|y_{-s+1}^{0}\right\|>R \Rightarrow \sum_{x_{1} \in E} p_{1, \theta}\left(x_{1} \mid x_{0}, y_{-s+1}^{0}\right)\left\|\Lambda^{\left(x_{1}\right)}\right\|^{2}<1 .
$$

This is true in particular if

$$
\forall x \in E,\left\|\Lambda^{(x)}\right\|<1 .
$$

The model fitted to the lynx data in the previous section satisfies condition (3.8) for the matrix norm defined as

$$
\|A\|=\left\|P^{-1} A P\right\|_{\infty},
$$

with $P$ the matrix containing the eigenvectors of the companion matrix for the second regime and $\|\cdot\|_{\infty}$ the infinity norm. This condition implies that all the regimes are stable. However, it is also possible to construct models which satisfy (3.7) with some unstable regimes if the instability is controlled by the dynamics of $\left\{X_{k}\right\}$.

Remark 3.3. The results given in this section are still valid when

- the noise $\{\epsilon\}_{k}$ in (3.2) is an iid sequence with finite variance which admits a pdf $f$ with respect to the Lebesgue measure such that for all $R>0, \inf _{y \in E_{R}} f(y)>0$, and

- $E=\{1, \ldots, M\}$ with $M \geq 2$ and (3.3) replaced by any transition kernel $p_{1, \theta}$ satisfying (2.1).

\subsection{Consistency of MLE}

The results given in this section generalize the results given in $[12,16]$ for homogeneous MS-AR models with linear Gaussian autoregressive models.

Corollary 3.4. Assume that Hypotheses 2 and 3 hold true for every $\theta$. Let $\Theta$ be a compact subset of $\tilde{\Theta}$. Then, for all $\theta \in \Theta$ there exists a unique invariant probability distribution and, for every $x_{0} \in M$ and every initial probability distribution $\nu$, the limit values of $\left(\hat{\theta}_{n, x_{0}}\right)_{n}$ are $\overline{\mathbb{P}}_{\theta^{*}}$-almost surely contained in $\left\{\theta \in \Theta: \overline{\mathbb{P}}_{\theta}=\overline{\mathbb{P}}_{\theta^{*}}\right\}$. 
Proof. This corollary is a direct consequence of Theorem 2.1 and of the previous section. As already noticed in Section 2, the invariant measure has a positive pdf with respect to $\mu$. As seen in the previous section, the Markov chain is aperiodic positive Harris recurrent (which implies (2.5)) and the stationary process is square integrable, which implies (2.2) and (2.3). In this example, $p_{2, \theta}$ is bounded from above and so (2.4) holds.

Remark 3.5. Corollary 3.4 is still valid when $E=\{1, \ldots, M\}$ with $M \geq 2$ and (3.3) replaced by any transition kernel $p_{1, \theta}$ satisfying (2.1).

In the sequel, we explicit the limit set $\left\{\theta \in \Theta: \overline{\mathbb{P}}_{\theta}=\overline{\mathbb{P}}_{\theta^{*}}\right\}$ under the supplementary condition

$$
\left(\beta_{0}^{(1)}, \beta_{1}^{(1)}, \ldots, \beta_{s}^{(1)}, \sigma^{(1)}\right) \neq\left(\beta_{0}^{(2)}, \beta_{1}^{(2)}, \ldots, \beta_{s}^{(2)}, \sigma^{(2)}\right),
$$

that the dynamics in the two regimes are distinct. Note that this condition is not sufficient in order to ensure identifiability. First, it can be easily seen that the homogeneous MS-AR model can be written in many different ways using the parametrization (3.3). It led us to add one of the following constraints on the parameters

$$
\forall x \in\{1,2\}, \quad \lambda_{1}^{(x)} \neq 0,
$$

which does not include the homogeneous model as a particular case or

$$
\forall x \in\{1,2\}, \pi_{-}^{(x)}=\pi_{+}^{(x)}=\pi_{0} \text { where } 0<\pi_{0}<1 / 2 \text { is a fixed constant, }
$$

in order to solve this problem. A practical motivation for (3.11) is given in Section 3.1. Let $\Theta^{\prime}$ be the set of $\theta \in \tilde{\Theta}$ satisfying (3.10) and let $\Theta^{\prime \prime}$ be the set of $\theta \in \tilde{\Theta}$ satisfying (3.11). Then, a permutation of the two states also leads different parameters values but to the same model. This problem can be solved by ordering the regimes or by allowing a permutation of the states as discussed below.

Proposition 3.6 (Identifiability). Let $\theta_{1}$ and $\theta_{2}$ belong to $\Theta^{\prime}\left(\right.$ resp. $\left.\Theta^{\prime \prime}\right)$ with $\theta_{i}=\left(\theta_{i}^{(1)}, \theta_{i}^{(2)}\right)$ and

$$
\theta_{i}^{(x)}=\left(\left(\beta_{j,(i)}^{(x)}\right)_{j \in\{0, \ldots s\}}, \sigma_{i},\left(\lambda_{j,(i)}^{(x)}\right)_{j \in\{0,1\}}\right),
$$

the parameters associated with the regime $x \in\{1,2\}$.

Assume that $\theta_{1}$ satisfies (3.9). Then $\overline{\mathbb{P}}_{\theta_{1}}^{Y}=\overline{\mathbb{P}}_{\theta_{2}}^{Y}$ if and only if $\theta_{1}$ and $\theta_{2}$ define the same model up to a permutation of indices, i.e. there exists a permutation $\tau$ of $\{1,2\}$ such that $\theta_{1}^{(x)}=\theta_{2}^{(\tau(x))}$.

The proof of Proposition 3.6 is postponed to Appendix B.

Now due to Corollary 3.4 and Proposition 3.6, we directly get Theorem 3.7.

Theorem 3.7. Assume that Hypotheses 2 and 3 hold true for every $\theta$. Let $\Theta$ be a compact subset of $\Theta^{\prime}$ or $\Theta^{\prime \prime}$. Assume that $\theta^{*}$ satisfies (3.9). Then, for every $x_{0} \in\{1,2\}$ and any initial probability distribution $\nu$, on a set of probability one, the limit values $\theta$ of the sequence of random variables $\left(\hat{\theta}_{n, x_{0}}\right)_{n}$ are equal to $\theta^{*}$ up to a permutation of indices.

\section{Non-Homogeneous Hidden Markov MODELS With EXogenous VARIABLES}

\subsection{Model}

When using NHMS-AR models in practice, it is often assumed that the evolution of $\left\{X_{k}\right\}$ depends not only on lagged values of the process of interest but also on strictly exogenous variables. In order to handle such situation, we will denote $Y_{k}=\left(Z_{k}, R_{k}\right)$ with $\left\{Z_{k}\right\}$ the time series of covariates and $\left\{R_{k}\right\}$ the output time series to be modeled. Besides Hypothesis 1, various supplementary conditional independence assumptions can be made 
for specific applications. For example, in [14] it is assumed that the switching probabilities of $\left\{X_{k}\right\}$ only depend on the exogenous covariates

$$
p_{1, \theta}\left(x_{k} \mid x_{k-1}, r_{k-s}^{k-1}, z_{k-s}^{k-1}\right)=p_{1, \theta}\left(x_{k} \mid x_{k-1}, z_{k-1}\right),
$$

that the evolution of $\left\{Z_{k}\right\}$ is independent of $\left\{X_{k}\right\}$ and $\left\{R_{k}\right\}$ and that $R_{k}$ is conditionally independent of $Z_{k-s}^{k}$ and $R_{k-s}^{k-1}$ given $X_{k}$

$$
p_{2, \theta}\left(z_{k}, r_{k} \mid x_{k}, z_{k-s}^{k-1}, r_{k-s}^{k-1}\right)=p_{R, \theta}\left(r_{k} \mid x_{k}\right) p_{Z}\left(z_{k} \mid z_{k-1}\right) .
$$

This model is often referred to as Non-Homogeneous Hidden Markov models (NHMMs) in the literature. The DAG below summarizes its dependence structure when $s=1$.

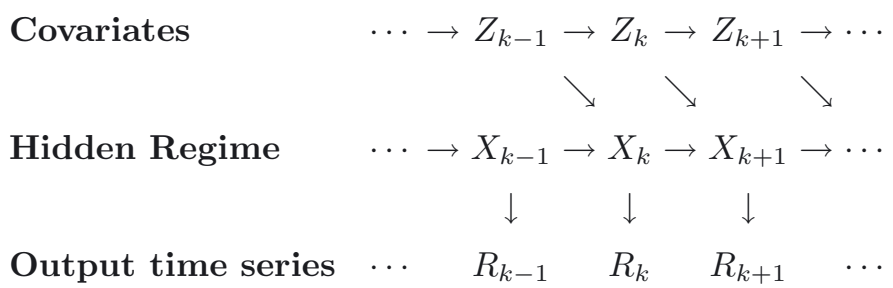

In this section, we consider a typical example of NHMM with finite hidden state space and strictly exogenous variables $\left(Z_{k}\right.$ does not include lagged values of $\left.\left\{R_{l}\right\}\right)$ and show that the theoretical results proven in this paper apply to this model. We focus on a model initially introduced in [5] for downscaling rainfall. It is an extension of the model proposed in [14] (see also [25] for more recent references). The results given in this section can be adapted to other NHMM with finite hidden state space such as the one proposed in [8], which is widely used in econometrics. The model is described more precisely hereafter.

Hypothesis 4. Let $M$ be a positive integer and $\Sigma$ be a $m \times m$ positive definite symmetric matrix. We suppose that $E=\{1, \ldots, M\}$ (endowed with the counting measure $\mathfrak{m}_{E}$ on $E$ ) and that the observed process has two components $Y_{k}=\left(Z_{k}, R_{k}\right)$. For every time $k, Z_{k} \in \mathcal{Z} \subseteq \mathbb{R}^{m}$ is a vector of $m$ large scale atmospheric variables (covariates) and $R_{k} \in\left(\left[0,+\infty[)^{\ell}\right.\right.$ is the daily accumulation of rainfall measured at $\ell$ meteorological stations (output time series) with the value 0 corresponding to dry days. The model aims at describing the conditional distribution of $\left\{R_{k}\right\}$ given $\left\{Z_{k}\right\}$. For this, we assume that

$$
p_{1, \theta}\left(x_{k} \mid x_{k-1}, y_{k-1}\right)=\frac{q_{x_{k-1}, x_{k}} \exp \left(-1 / 2\left(z_{k-1}-\mu_{x_{k-1}, x_{k}}\right)^{\prime} \Sigma^{-1}\left(z_{k-1}-\mu_{x_{k-1}, x_{k}}\right)\right)}{\sum_{x^{\prime \prime}=1}^{M} q_{x_{k-1}, x "} \exp \left(-1 / 2\left(z_{k-1}-\mu_{x_{k-1}, x^{\prime \prime}}\right)^{\prime} \Sigma^{-1}\left(z_{k-1}-\mu_{x_{k-1}, x^{\prime \prime}}\right)\right)},
$$

with $q_{x, x^{\prime}}>0, \mu_{x, x^{\prime}} \in \mathbb{R}^{m}$ and (4.1) holds with respect to $\mathfrak{m}_{\mathcal{Z}} \otimes \mathfrak{m}_{0}^{\otimes \ell}$, where $\mathfrak{m}_{\mathcal{Z}}$ is the Lebesgue's measure on $\mathcal{Z}$ and where $\mathfrak{m}_{0}$ is the sum of the Dirac's measure $\delta_{0}$ and of the Lebesgue measure on $(0,+\infty[$. We observe that $\left\{Z_{k}\right\}_{k}$ is a Markov chain whose transition kernel depends neither on the current weather type nor on the unknown parameter $\theta$ (typically $Z_{k}$ is the output of an atmospheric model and is considered as an input to the Markov switching model) and that the conditional distribution of $R_{k}$ given $X_{k}$ and $\left\{Y_{k^{\prime}}\right\}_{k^{\prime}<k}$ only depends on $X_{k}$ as in usual HMMs. Finally the rainfall at the different locations is assumed to be conditionally independent given the weather type

$$
p_{R, \theta}\left(r_{k}(1), \ldots, r_{k}(l) \mid x_{k}\right)=\prod_{i=1}^{\ell} p_{R_{i}, \theta}\left(r_{k}(i) \mid x_{k}\right),
$$

and the rainfall at the different locations is given by the product of Bernoulli and Gamma's variables

$$
p_{R_{i}, \theta}\left(r_{k}(i) \mid x_{k}\right)= \begin{cases}1-\pi_{i}^{\left(x_{k}\right)} & \left(r_{k}(i)=0\right) \\ \pi_{i}^{\left(x_{k}\right)} \gamma\left(r_{k}(i) ; \alpha_{i}^{\left(x_{k}\right)}, \beta_{i}^{\left(x_{k}\right)}\right) & \left(r_{k}(i)>0\right),\end{cases}
$$


where $0<\pi_{i}^{(x)}<1, \alpha_{i}^{(x)}>0, \beta_{i}^{(x)}>0$ and $\gamma(. ; \alpha, \beta)$ denotes the pdf of a Gamma distribution with parameters $\alpha, \beta$ :

$$
\gamma(r ; \alpha, \beta)=r^{\alpha-1} \frac{\beta^{\alpha} e^{-\beta r}}{\Gamma(\alpha)} .
$$

The parameter $\theta$ corresponds to

$$
\theta=\left(\left(q_{x, x^{\prime}}\right),\left(\mu_{x, x^{\prime}}\right),\left(\pi_{i}^{(x)}\right),\left(\alpha_{i}^{(x)}\right),\left(\beta_{i}^{(x)}\right)\right) .
$$

We write $\tilde{\Theta}$ for the set of such parameters $\theta$ satisfying, for every $x \in\{1, \ldots, M\}$ and every $i \in\{1, \ldots, \ell\}$,

$$
\sum_{x^{\prime}=1}^{M} q_{x, x^{\prime}}=1, \quad 0<q_{x, x^{\prime}}<1, \sum_{x^{\prime}=1}^{M} \mu_{x, x^{\prime}}=0,0<\pi^{(x)}<1, \alpha_{i}^{(x)}>0, \text { and } \beta_{i}^{(x)}>0 .
$$

The conditions $\sum_{x^{\prime}=1}^{M} q_{x, x^{\prime}}=1$ and $\sum_{x^{\prime}=1}^{M} \mu_{x, x^{\prime}}=0$ come from [14]. These conditions are not restrictive. Indeed, $q_{\theta}$ is unchanged if we replace $\mu_{x, x^{\prime}}$ by $\mu_{x, x^{\prime}}-\sum_{x}, \mu_{x, x^{\prime \prime}}$ and $q_{x, x^{\prime}}$ by $\frac{q_{x, x^{\prime}} \exp \left(-\left(\mu_{x, x^{\prime}}\right) \Sigma^{-1} \mu_{x}\right)}{\sum_{x^{\prime \prime}} q_{x, x^{\prime \prime}} \exp \left(-\left(\mu_{x, x^{\prime \prime}}\right) \Sigma^{-1} \mu_{x}\right)}$ (with $\left.\mu_{x}:=\sum_{x "} \mu_{x, x}\right)$.

Observe that the fact that, if $\mu_{x, x^{\prime}}=0$ for every $x, x^{\prime}$, then $\left\{X_{k}\right\}_{k}$ is an homogeneous Markov chain and $\left\{Z_{k}\right\}_{k}$ does not plays any role in the dynamics of $\left\{X_{k}, R_{k}\right\}_{k}$.

\subsection{Properties of the Markov chain}

We start by recalling a classical result ensuring (2.5) in the context of HMM (a proof of this result is given in Appendix D for completeness).

Lemma 4.1 (HMM). Fix $\theta$. Assume that $p_{1, \theta}\left(x \mid x^{\prime}, y^{\prime}\right)=p_{1, \theta}\left(x \mid x^{\prime}\right)$ does not depend on $y^{\prime},\left\{X_{k}\right\}_{k}$ is a Markov chain with transition kernel $Q_{1, \theta}$ admitting a unique invariant probability measure with pdf $h_{1, \theta}$ (wrt $\mathfrak{m}_{E}$ ) such that

$$
\lim _{n \rightarrow+\infty} \sup _{\nu \in \mathcal{P}(E)}\left\|\left[Q_{1, \theta}^{* n} \nu\right]-h_{1, \theta}\right\|_{L^{1}\left(\mathfrak{m}_{E}\right)}=0 .
$$

Assume moreover that $p_{2, \theta}\left(y \mid x, y^{\prime}\right)=p_{2, \theta}(y \mid x)$. Then there exists a unique invariant measure $\nu_{\theta}$ with $p d f h_{\theta}$ $\left(\right.$ wrt $\left.\mathfrak{m}_{E} \times \mathfrak{m}_{K}\right)$ given by $h_{\theta}(x, y):=h_{1, \theta}(x) p_{2, \theta}(y \mid x)$ and

$$
\lim _{n \rightarrow+\infty} \sup _{\nu \in \mathcal{P}(E \times K)}\left\|\left[Q_{\theta}^{* n} \nu\right]-h_{\theta}\right\|_{L^{1}\left(\mathfrak{m}_{E} \times \mathfrak{m}_{K}\right)}=0 .
$$

Moreover, if $p_{2, \theta}>0$ and if $\left\{X_{k}\right\}_{k}$ is an aperiodic positive Harris recurrent Markov chain, then the Markov chain $\left\{X_{k}, Y_{k}\right\}_{k}$ is positive Harris recurrent and aperiodic.

Due to this lemma, assumption (2.5) holds true and $\left\{X_{k}, Y_{k}\right\}_{k}$ is aperiodic positive Harris recurrent as soon as $\left\{X_{k}, Z_{k}\right\}_{k}$ is aperiodic positive Harris recurrent.

\subsection{Consistency of MLE}

Corollary 4.2. Assume Hypothesis 4. Assume that $\Theta$ is a compact subset of $\tilde{\Theta}$ and that, for every $\theta \in \Theta$, the transition kernel $Q_{0, \theta}$ of the Markov chain $\left\{X_{k}, Z_{k}\right\}_{k}$ admits a unique invariant probability measure with pdf $h_{0, \theta}>0\left(\right.$ wrt $\left.\mathfrak{m}_{E} \times \mathfrak{m}_{\mathcal{Z}}\right)$ such that

$$
\lim _{n \rightarrow+\infty} \sup _{\nu \in \mathcal{P}(E \times \mathcal{Z})}\left\|\left[Q_{0, \theta}^{* n} \nu\right]-h_{0, \theta}\right\|_{L^{1}\left(\mathfrak{m}_{E} \times \mathfrak{m}_{\mathcal{Z}}\right)}=0 .
$$

Assume moreover that $\mathcal{Z}$ is compact, that

$$
\forall z \in \mathcal{Z}, \quad \sup _{z_{-1} \in \mathcal{Z}} p_{Z}\left(z \mid z_{-1}\right)<\infty
$$


and that

$$
\overline{\mathbb{E}}_{\theta^{*}}\left[\left|\log p_{Z}\left(Z_{0} \mid Z_{-1}\right)\right|\right]<\infty .
$$

Then, for every $x_{0} \in\{1, \ldots, M\}$, on a set of probability one (for $\left.\overline{\mathbb{P}}_{\theta^{*}}\right)$, the limit values $\theta$ of the sequence of random variables $\left(\hat{\theta}_{n, x_{0}}\right)_{n}$ are $\overline{\mathbb{P}}_{\theta^{*}}$-almost surely contained in $\left\{\theta \in \Theta: \overline{\mathbb{P}}_{\theta}=\overline{\mathbb{P}}_{\theta^{*}}\right\}$.

If, moreover, $\left\{X_{k}, Z_{k}\right\}_{k}$ is aperiodic and positive Harris recurrent then this result holds true for any initial probability distribution.

Proof. Due to the previous section, we know that (4.3) implies (2.5) and that the aperiodicity and positive Harris recurrence of $\left\{X_{k}, Z_{k}\right\}_{k}$ implies the positive Harris recurrence of $\left\{X_{k}, Y_{k}\right\}_{k}$.

The fact that $\Theta$ is a compact subset of $\tilde{\Theta}$ directly implies (2.1).

Assumption (2.4) holds true since $E$ is finite, since $p_{R, \theta}(r \mid x)<\infty$ for every $(x, y) \in E \times K$ and according to $(4.4)$.

Now according to (4.5), (2.2) and (2.3) will follow from the fact that, for every $x_{0} \in X$ and every $i \in\{1, \ldots, \ell\}$,

$$
\overline{\mathbb{E}}_{\theta^{*}}\left[\left|\log \left(\inf _{\theta} p_{R_{i}, \theta}\left(R_{i} \mid x_{0}\right)\right)\right|\right]+\overline{\mathbb{E}}_{\theta *}\left[\left|\log \left(\sup _{\theta} p_{R_{i}, \theta}\left(R_{i} \mid x_{0}\right)\right)\right|\right]<\infty .
$$

Now we observe that if $R_{i}=0$, then

$$
0<1-\pi_{+} \leq p_{R_{i}, \theta}\left(R_{i} \mid x_{0}\right) \leq 1-\pi_{-},
$$

where $\pi_{-}$and $\pi_{+}$are the minimal and maximal possible values of $\pi_{i}^{(x)}$ (for $x \in X, i \in\{1, \ldots, \ell\}$ and $\theta$ in the compact set $\Theta$ ). Analogously, let us write $\alpha_{-}, \alpha_{+}$for the minimal and maximal possible values of $\alpha_{i}^{(x)}$ and $\beta_{-}$, $\beta_{+}$for the minimal and maximal possible values of $\beta_{i}^{(x)}$. Since, all this quantities are positive and finite, due to the expression of $\log \left(p_{R_{i}, \theta}\left(R_{i} \mid x_{0}\right)\right)$, to prove $(2.2)$ and (2.3), it is enough to prove that

$$
\overline{\mathbb{E}}_{\theta^{*}}\left[R_{i}\right]<\infty \text { and } \overline{\mathbb{E}}_{\theta^{*}}\left[\left|\log \left(R_{i}\right)\right| \mathbf{1}_{\left\{R_{i}>0\right\}}\right]<\infty .
$$

Observe that, under the stationary distribution, the pdf $h_{i}$ of $R_{i}$ satisfies:

$$
\forall r>0, \quad h_{i}(r) \leq\left(r^{\alpha_{-}-1} \mathbf{1}_{\{r \leq 1\}}+r^{\alpha_{+}-1} \mathbf{1}_{\{r>1\}}\right) \frac{\max \left(\beta_{+}^{\alpha_{+}}, \beta_{+}^{\alpha_{-}}\right) e^{-r \beta_{-}}}{\Gamma\left(\alpha_{-}\right)} .
$$

Therefore, (2.2) and (2.3) come from the facts that $r \mapsto r^{\alpha_{+}-1} e^{-r \beta_{-}}$is integrable at $+\infty$ (since $\left.\beta_{-}>0\right)$ and that $r \mapsto|\log r| r^{\alpha_{-}-1}$ is integrable at 0 (since $\alpha_{-}>0$ ).

Now we will add an assumption on $\theta$ to ensure the identifiability of the parameter. If we assume $\pi_{i}^{(x)}=0$ for every $i$ and every $x$, then identifiability follows easily if we assume moreover that

$$
x \neq x^{\prime} \Rightarrow\left(\alpha_{i}^{(x)}, \beta_{i}^{(x)}\right)_{i} \neq\left(\alpha_{i}^{\left(x^{\prime}\right)}, \beta_{i}^{\left(x^{\prime}\right)}\right)_{i} .
$$

But, if we do not assume $\pi_{i}^{(x)}=0,(4.6)$ does not ensure identifiability anymore. We give now an explicit counter-example.

Remark 4.3. Assume $M=\ell=2$. We consider two models $A_{1}$ and $A_{2}$ associated to $\theta_{1}$ and $\theta_{2}$, respectively, with

$$
\theta_{j}=\left(\left(q_{x, x^{\prime},(j)}\right),\left(\mu_{x, x^{\prime},(j)}\right),\left(\pi_{i}^{(x,(j))}\right),\left(\alpha_{i}^{(x,(j))}\right),\left(\beta_{i}^{(x,(j))}\right)\right),
$$

and

$$
\text { - } q_{x, x^{\prime},(1)}=0.5, \mu_{x, x^{\prime},(1)}=0, \pi_{i}^{(x,(1))}=0.5, \alpha_{i}^{(x,(1))}=1, \beta_{1}^{(x,(1))}=1, \beta_{2}^{(1,(1))}=2, \beta_{2}^{(2,(1))}=3 \text {, }
$$


- $q_{x, 1,(2)}=0.6, q_{x, 2,(2)}=0.4, \mu_{x, x^{\prime},(2)}=0, \pi_{1}^{(x,(2))}=0.5, \pi_{2}^{(1,(2))}=\frac{0.25}{0.6}, \pi_{2}^{(2,(2))}=\frac{0.25}{0.4}, \alpha_{i}^{(x,(2))}=1$, $\beta_{1}^{(x,(1))}=1, \beta_{2}^{(1,(1))}=2, \beta_{2}^{(2,(1))}=3$.

For model $A_{1}$ (under the stationary measure), $\left\{X_{k}\right\}$ is an iid sequence on $\{1,2\}$ with $\mathbb{P}\left(X_{1}=1\right)=0.5$ and the distribution of $R_{k}$ given $\left\{X_{k}=1\right\}$ is $\left(0.5 \delta_{0}+0.5 \Gamma(1,1)\right) \otimes\left(0.5 \delta_{0}+0.5 \Gamma(1,2)\right)$ whereas the distribution of $R_{k}$ taken $\left\{X_{k}=2\right\}$ is $\left(0.5 \delta_{0}+0.5 \Gamma(1,1)\right) \otimes\left(0.5 \delta_{0}+0.5 \Gamma(1,3)\right)$. Hence, for the model $A_{1}$, the $R_{k}$ are iid with distribution

$$
\left(0.5 \delta_{0}+0.5 \Gamma(1,1)\right) \otimes\left(0.5 \delta_{0}+0.25 \Gamma(1,2)+0.25 \Gamma(1,3)\right)
$$

For model $A_{2}$ (under the stationary measure), $\left\{X_{k}\right\}$ is an iid sequence on $\{1,2\}$ with $\mathbb{P}\left(X_{1}=1\right)=0.6$ and the distribution of $R_{k}$ given $\left\{X_{k}=1\right\}$ is $\left(0.5 \delta_{0}+0.5 \Gamma(1,1)\right) \otimes\left(\left(1-\frac{0.25}{0.6}\right) \delta_{0}+\frac{0.25}{0.6} \Gamma(1,2)\right)$ whereas the distribution of $R_{k}$ taken $\left\{X_{k}=2\right\}$ is $\left(0.5 \delta_{0}+0.5 \Gamma(1,1)\right) \otimes\left(\left(1-\frac{0.25}{0.4}\right) \delta_{0}+\frac{0.25}{0.4} \Gamma(1,3)\right)$. Hence, for the model $A_{2}$, the $R_{k}$ are iid with distribution (4.7).

Observe that the distribution of $\left\{Y_{k}\right\}$ under the stationary measure is the same for models $A_{1}$ and $A_{2}$.

The next result (proved in Appendix C) states that the following condition ensures identifiability

$$
x \neq x^{\prime} \Rightarrow \forall i \in\{1, \ldots, \ell\},\left(\alpha_{i, \theta_{1}}^{(x)}, \beta_{i, \theta_{1}}^{(x)}\right)=\left(\alpha_{i, \theta_{1}}^{\left(x^{\prime}\right)}, \beta_{i, \theta_{1}}^{\left(x^{\prime}\right)}\right) .
$$

Proposition 4.4. Assume Hypothesis 4. Let $\theta_{1}$ and $\theta_{2}$ in $\tilde{\Theta}$, with

$$
\theta_{j}=\left(\left(q_{x, x^{\prime},(j)}\right),\left(\mu_{x, x^{\prime},(j)}\right),\left(\pi_{i}^{(x,(j))}\right),\left(\alpha_{i}^{(x,(j))}\right),\left(\beta_{i}^{(x,(j))}\right)\right) .
$$

Assume that $\theta_{1}$ satisfies (4.8).

Then $\overline{\mathbb{P}}_{\theta_{1}}^{Y}=\overline{\mathbb{P}}_{\theta_{2}}^{Y}$ if and only $\theta_{1}$ and $\theta_{2}$ are equal up to a permutation of indices, i.e. there exists a permutation $\tau$ of $\{1, \ldots, M\}$ such that, for every $x, x^{\prime} \in\{1, \ldots, M\}$ and every $i \in\{1, \ldots, \ell\}$, we have $q_{x, x^{\prime},(1)}=q_{\tau(x), \tau\left(x^{\prime}\right),(2)}$, $\mu_{x, x^{\prime},(1)}=\mu_{\tau(x), \tau\left(x^{\prime}\right),(2)}, \pi_{i}^{(x,(1))}=\pi_{i}^{(\tau(x),(2))}, \alpha_{i}^{(x,(1))}=\alpha_{i}^{(\tau(x),(j))}, \beta_{i}^{(x,(1))}=\beta_{i}^{(\tau(x),(2))}$.

Now the following result is a direct consequence of Corollary 4.2 and Proposition 4.4.

Theorem 4.5. Assume Hypothesis 4. Assume that $\Theta$ is a compact subset of $\tilde{\Theta}$ and that, for every $\theta \in \Theta$, the transition kernel $Q_{0, \theta}$ of the Markov chain $\left(X_{k}, Z_{k}\right)_{k}$ admits a unique invariant probability measure with pdf $h_{0, \theta}$ (wrt $\mathfrak{m}_{E} \times \mathfrak{m}_{\mathcal{Z}}$ ) satisfying (4.3). Assume that $\theta^{*}$ satisfies (4.8). Assume moreover that $\mathcal{Z}$ is compact, that (4.4) and (4.5) hold true. Then, for every $x_{0} \in\{1, \ldots, M\}$, on a set of probability one (for $\overline{\mathbb{P}}_{\theta^{*}}$ ), the limit values $\theta$ of the sequence of random variables $\left(\hat{\theta}_{n, x_{0}}\right)_{n}$ are equal to $\theta^{*}$ up to a permutation of indices.

If, moreover, $\left(X_{k}, Z_{k}\right)_{k}$ is aperiodic and positive Harris recurrent then this result holds true for any initial probability distribution.

\section{Conclusions}

In this work, we have extended the consistency result of [10] to the non-homogeneous case and we have relaxed some other of their assumptions (namely on $p_{2}$ ). We have illustrated our results by two specific but representative models for which we gave general conditions ensuring the consistency of the maximum likelihood estimator. Our results opens perspectives in different directions: theoretical results (such as the asymptotic normality of the MLE), applied statistics (namely the study of other non-homogeneous switching Markov models and their applications), but also the development of a $\mathrm{R}$ package to make easier the practical use of these flexible models. 


\section{Appendix A. Consistency: Proof of Theorem 2.1}

As usual, we define the associated transition operator $Q_{\theta}$ as an operator acting on the set of bounded measurable functions of $E \times K^{s}$ (it may also act on other Banach spaces $\mathcal{B}$ ) by

$$
\begin{aligned}
Q_{\theta} g\left(x_{0}, y_{-s+1}^{0}\right) & =\mathbb{E}_{\theta}\left[g\left(X_{1}, Y_{-s+2}^{1}\right) \mid X_{0}=x_{0}, Y_{-s+1}^{0}=y_{-s+1}^{0}\right] \\
& =\int_{E \times K} g\left(x_{1}, y_{-s+2}^{1}\right) q_{\theta}\left(x_{1}, y_{1} \mid x_{0}, y_{-s+1}^{0}\right) d \mu_{0}\left(x_{1}, y_{1}\right) .
\end{aligned}
$$

We denote by $Q_{\theta}^{*}$ the adjoint operator of $Q_{\theta}$ defined on $\mathcal{B}^{\prime}$ the dual space of $\mathcal{B}$ (if $Q_{\theta}$ acts on $\mathcal{B}$ ) by

$$
\forall \nu \in \mathcal{B}^{\prime}, \forall f \in \mathcal{B}, \quad Q_{\theta}^{*}(\nu)(f)=\nu\left(Q_{\theta}(f)\right) .
$$

For every integer $k \geq 0$, the measure $\left(Q_{\theta}^{*}\right)^{k}(\nu)$ corresponds to the distribution of $\left(X_{k}, Y_{k-s+1}^{k}\right)$ if $\left\{X_{l}, Y_{l}\right\}_{l}$ is the Markov chain with transition operator $Q_{\theta}$ such that the distribution of $\left(X_{0}, Y_{-s+1}^{0}\right)$ is $\nu$.

If $\nu \in \mathcal{B}^{\prime}$ has a pdf $h$ with respect to $\mu:=\mathfrak{m}_{E} \times \mathfrak{m}_{K}^{\otimes s}$, then $Q_{\theta}^{*} \nu$ is also absolutely continuous with respect to $\mu$ and its pdf, written $Q_{\theta}^{*} h$, is given by

$$
Q_{\theta}^{*} h\left(x_{0}, y_{-s+1}^{0}\right):=\int_{E \times K} q_{\theta}\left(x_{0}, y_{0} \mid x_{-1}, y_{-s}^{-1}\right) h\left(x_{-1}, y_{-s}^{-1}\right) d \mu_{0}\left(x_{-1}, y_{-s}\right) .
$$

Observe that, due to the particular form of $q_{\theta}$, for every integer $k \geq s$ and every $P=\left(x_{-k}, y_{-k-s+1}^{-k}\right) \in E \times K^{s}$, the measure $\left(Q_{\theta}^{*}\right)^{k} \delta_{P}$ (where $\delta_{P}$ is the Dirac measure at $P$ ) is absolutely continuous with respect to $\mu:=$ $\mathfrak{m}_{E} \times \mathfrak{m}_{K}^{\otimes s} ;$ its pdf $Q_{\theta}^{* k}(\cdot \mid P)$ is given by

$$
Q_{\theta}^{* k}\left(x_{0}, y_{-s+1}^{0} \mid P\right)=\int_{E^{k-1} \times K^{k-s}} \prod_{i=1-k}^{0} q_{\theta}\left(x_{i}, y_{i} \mid x_{i-1}, y_{i-s}^{i-1}\right) d \mathfrak{m}_{E}^{\otimes(k-1)}\left(x_{-k+1}^{-1}\right) d \mathfrak{m}_{K}^{\otimes(k-s)}\left(y_{-k+1}^{-s}\right) .
$$

More generally, for every initial measure $\nu$ and every $k \geq s, Q_{\theta}^{* k} \nu$ is absolutely continuous with respect to $\mu$ and its pdf $\left[Q_{\theta}^{* k} \nu\right]$ is given by

$$
\left[Q_{\theta}^{* k} \nu\right](\cdot)=\int_{E \times K^{s}} Q_{\theta}^{* k}(\cdot \mid P) d \nu(P) .
$$

We suppose that, for every $\theta \in \Theta$, there exists an invariant probability measure $\bar{\nu}_{\theta}$ for $Q_{\theta}^{*}$. Observe that, due to (A.1), $\bar{\nu}_{\theta}$ admits a pdf $h_{\theta}$ with respect to $\mu$.

We identify $\left(X_{k}, Y_{k}\right)_{k}$ with the canonical Markov chain $\left\{\left(X_{0}, Y_{0}\right) \circ \tau^{k}\right\}_{k}$ defined on $\Omega_{+}:=(E \times K)^{\mathbb{N}}$ by $X_{0}\left(\left(x_{k}, y_{k}\right)_{k}\right)=x_{0}, Y_{0}\left(\left(x_{k}, y_{k}\right)_{k}\right)=y_{0}, \tau_{+}$being the shift $\left(\tau_{+}\left(\left(x_{k}, y_{k}\right)_{k}\right)=\left(x_{k+1}, y_{k+1}\right)_{k}\right)$. We endow $\Omega_{+}$with its Borel $\sigma$-algebra $\mathcal{F}_{+}$. We denote by $\overline{\mathbb{P}}_{\theta}$ the probability measure on $\left(\Omega_{+}, \mathcal{F}_{+}\right)$associated to the invariant measure $\bar{\nu}_{\theta}$ and by $\overline{\mathbb{E}}_{\theta}$ the corresponding expectation.

We now follow and adapt the proof of [10], Theorem 1 (see Lems. A.8 and A.9). We do not give all the details of the proofs when they are a direct rewriting of [10]. First, we consider the stationary case. Let $\tau$ be the full shift on $\Omega:=(E \times K)^{\mathbb{Z}}$. For every $k \in \mathbb{Z}$, we identify $X_{k}$ with $X_{0} \circ \tau^{k}$ and $Y_{k}$ with $Y_{0} \circ \tau^{k}$, where $X_{0}\left(\left(x_{m}, y_{m}\right)_{m \in \mathbb{Z}}\right):=x_{0}$ and $Y_{0}\left(\left(x_{m}, y_{m}\right)_{m \in \mathbb{Z}}\right)=y_{0}$.

\section{A.1. Likelihood and stationary likelihood}

We start by recalling a classical fact in the context of Markov chains (and the proof of which is direct).

Fact A.1. Let $m$ and $n$ belong to $\mathbb{Z}$ with $m \leq n$. Under $\overline{\mathbb{P}}_{\theta}$, conditionally to $\left(Y_{m-s+1}^{n}\right),\left(X_{k}\right)_{k \in\{m, \ldots, n\}}$ is a (possibly nonhomogeneous) Markov chain. Moreover, under $\overline{\mathbb{P}}_{\theta}$, the conditional pdf (wrt $\mathfrak{m}_{E}$ ) of $X_{k}$ given $\left(X_{m}^{k-1}, Y_{m-s+1}^{n}\right)$ is given by

$$
p_{\theta}\left(X_{k}=x_{k} \mid X_{m}^{k-1}, Y_{m-s+1}^{n}\right)=\frac{p_{\theta}\left(Y_{k}^{n}, X_{k}=x_{k} \mid X_{k-1}, Y_{k-s}^{k-1}\right)}{p_{\theta}\left(Y_{k}^{n} \mid X_{k-1}, Y_{k-s}^{k-1}\right)} \overline{\mathbb{P}}_{\theta}-\text { a.s. },
$$


with

$$
p_{\theta}\left(Y_{k}^{n}, X_{k}=x_{k} \mid X_{k-1}=x_{k-1}, Y_{k-s}^{k-1}\right):=\int_{E^{n-k}} \prod_{j=k}^{n} q_{\theta}\left(x_{j}, Y_{j} \mid x_{j-1}, Y_{j-s}^{j-1}\right) d \mathfrak{m}_{E}^{\otimes(n-k)}\left(x_{k+1}^{n}\right),
$$

and

$$
p_{\theta}\left(Y_{k}^{n} \mid X_{k-1}, Y_{k-s}^{k-1}\right):=\int_{E} p_{\theta}\left(Y_{k}^{n}, X_{k}=x_{k} \mid X_{k-1}, Y_{k-s}^{k-1}\right) d \mathfrak{m}_{E}\left(x_{k}\right) .
$$

Using (2.1)-(2.3), we observe that the quantities appearing in this fact are well-defined. Due to Fact A.1, the quantity $\bar{p}_{\theta}\left(X_{k}=x_{k} \mid X_{k-1}, Y_{m-s+1}^{n}\right)$ is equal to

$$
\frac{\int_{E^{n-k+1}}\left(\prod_{j=k+1}^{n} a_{j}\right) p_{1, \theta}\left(\tilde{x}_{k} \mid X_{k-1}, Y_{k-s}^{k-1}\right) p_{2, \theta}\left(Y_{k} \mid \tilde{x}_{k}, Y_{k-s}^{k-1}\right) d \delta_{x_{k}}\left(\tilde{x}_{k}\right) d \mathfrak{m}_{E}^{\otimes(n-k)}\left(\tilde{x}_{k+1}^{n}\right)}{\int_{E^{n-k+1}}\left(\prod_{j=k+1}^{n} a_{j}\right) p_{1, \theta}\left(\tilde{x}_{k} \mid X_{k-1}, Y_{k-s}^{k-1}\right) p_{2, \theta}\left(Y_{k} \mid \tilde{x}_{k}, Y_{k-s}^{k-1}\right) d \mathfrak{m}_{E}^{\otimes(n-k+1)}\left(\tilde{x}_{k}^{n}\right)},
$$

with $a_{j}:=q_{\theta}\left(\tilde{x}_{j}, Y_{j} \mid \tilde{x}_{j-1}, Y_{j-s}^{j-1}\right)$. Therefore

$$
\bar{p}_{\theta}\left(X_{k}=x_{k} \mid X_{k-1}, Y_{m-s+1}^{n}\right) \geq \frac{p_{1,-}}{p_{1,+}} \beta\left(x_{k}\right), \text { with } \beta\left(x_{k}\right):=\frac{p_{\theta}\left(Y_{k}^{n} \mid X_{k}=x_{k}, Y_{k-s}^{k-1}\right)}{\int_{E} p_{\theta}\left(Y_{k}^{n} \mid X_{k}=\tilde{x}_{k}, Y_{k-s}^{k-1}\right) d \mathfrak{m}_{E}\left(\tilde{x}_{k}\right)} .
$$

From this last inequality (since $0<p_{1,-}<p_{1,+}<\infty$ ), we directly get the following (from [20]).

Corollary A.2. (as [10], Cor. 1) For all $m \leq k \leq n$ and every probability measures $\mathfrak{m}_{1}$ and $\mathfrak{m}_{2}$ on E, we have, $\overline{\mathbb{P}}_{\theta}-$ a.s.

$$
\left\|\int_{E} \overline{\mathbb{P}}_{\theta}\left(X_{k} \in \cdot \mid X_{m}=x_{m}, Y_{m-s+1}^{n}\right) d \mathfrak{m}_{1}\left(x_{m}\right)-\int_{E} \overline{\mathbb{P}}_{\theta}\left(X_{k} \in \cdot \mid X_{m}=x_{m}, Y_{m-s+1}^{n}\right) d \mathfrak{m}_{2}\left(x_{m}\right)\right\|_{T V} \leq \rho^{k-m},
$$

with $\rho:=1-\frac{p_{1,-}}{p_{1,+}}$.

Observe that the $\log$-likelihood $\ell_{n}\left(\theta, x_{0}\right)$ satisfies

$$
\ell_{n}\left(\theta, x_{0}\right)=\sum_{k=1}^{n} \log p_{\theta}\left(Y_{k} \mid X_{0}=x_{0}, Y_{-s+1}^{k-1}\right) \quad \overline{\mathbb{P}}_{\theta}-\text { a.s. }
$$

with

$$
\begin{aligned}
p_{\theta}\left(Y_{k} \mid X_{0}=x_{0}, Y_{-s+1}^{k-1}\right) & :=\frac{p_{\theta}\left(Y_{1}^{k} \mid X_{0}=x_{0}, Y_{-s+1}^{0}\right)}{p_{\theta}\left(Y_{1}^{k-1} \mid X_{0}=x_{0}, Y_{-s+1}^{0}\right)} \\
& =\int_{E^{2}} q_{\theta}\left(x_{k}, Y_{k} \mid x_{k-1}, Y_{k-s}^{k-1}\right) p_{\theta}\left(X_{k-1}=x_{k-1} \mid X_{0}=x_{0}, Y_{-s+1}^{k-1}\right) d \mathfrak{m}_{E}^{\otimes 2}\left(x_{k}, x_{k-1}\right) .
\end{aligned}
$$

Let us now define the stationary log-likelihood $\ell_{n}(\theta)$ by

$$
\ell_{n}(\theta):=\sum_{k=1}^{n} \log \bar{p}_{\theta}\left(Y_{k} \mid Y_{-s+1}^{k-1}\right),
$$

with

$$
\bar{p}_{\theta}\left(Y_{k} \mid Y_{-s+1}^{k-1}\right):=\int_{E^{2}} q_{\theta}\left(x_{k}, Y_{k} \mid x_{k-1}, Y_{k-s}^{k-1}\right) \bar{p}_{\theta}\left(X_{k-1}=x_{k-1} \mid Y_{-s+1}^{k-1}\right) d \mathfrak{m}_{E}^{\otimes 2}\left(x_{k}, x_{k-1}\right)
$$

and

$$
\bar{p}_{\theta}\left(X_{k-1}=x_{k-1} \mid Y_{-s+1}^{k-1}\right):=\int_{E} p_{\theta}\left(X_{k-1}=x_{k-1} \mid X_{0}=x_{0}, Y_{-s+1}^{k-1}\right) \bar{p}_{\theta}\left(X_{0}=x_{0} \mid Y_{-s+1}^{k-1}\right) d \mathfrak{m}_{E}\left(x_{0}\right) .
$$


Lemma A.3. (as [10], Lem. 2) We have

$$
\sup _{x_{0} \in E} \sup _{\theta \in \Theta}\left|\ell_{n}\left(\theta, x_{0}\right)-\ell_{n}(\theta)\right| \leq \frac{1}{(1-\rho)^{2}} \quad \overline{\mathbb{P}}_{\theta^{*}}-\text { a.s. }
$$

Proof. We have

$$
\begin{aligned}
\sup _{x_{0} \in E} \mid p_{\theta}\left(Y_{k} \mid X_{0}=x_{0}, Y_{-s+1}^{k-1}\right) & -\bar{p}_{\theta}\left(Y_{k} \mid Y_{-s+1}^{k-1}\right) \mid \\
\leq & p_{1,+} \int_{E^{3}} p_{2, \theta}\left(Y_{k} \mid x_{k}, Y_{k-s}^{k-1}\right) D\left(x_{k-1}, x_{0}, x\right) \bar{p}_{\theta}\left(X_{0}=x \mid Y_{-s+1}^{k-1}\right) d \mathfrak{m}_{E}^{\otimes 3}\left(x, x_{k-1}, x_{k}\right),
\end{aligned}
$$

with $D\left(x_{k-1}, x_{0}, x\right):=\left|p_{\theta}\left(X_{k-1}=x_{k-1} \mid X_{0}=x_{0}, Y_{-s+1}^{k-1}\right)-p_{\theta}\left(X_{k-1}=x_{k-1} \mid X_{0}=x, Y_{-s+1}^{k-1}\right)\right|$. Due to Corollary A.2, we have

$$
\left|p_{\theta}\left(Y_{k} \mid X_{0}=x_{0}, Y_{-s+1}^{k-1}\right)-\bar{p}_{\theta}\left(Y_{k} \mid Y_{-s+1}^{k-1}\right)\right| \leq p_{1,+} \rho^{k-1} \int_{E} p_{2, \theta}\left(Y_{k} \mid x_{k}, Y_{k-s}^{k-1}\right) d \mathfrak{m}_{E}\left(x_{k}\right)
$$

Since $\left|p_{\theta}\left(Y_{k} \mid X_{0}, Y_{-s+1}^{k-1}\right)\right|$ and $\left|p_{\theta}\left(Y_{k} \mid Y_{-s+1}^{k-1}\right)\right|$ are both larger than or equal to

$$
p_{1,-} \int_{E} p_{2, \theta}\left(Y_{k} \mid x_{k}, Y_{k-s}^{k-1}\right) d \mathfrak{m}_{E}\left(x_{k}\right),
$$

we obtain that

$$
\begin{aligned}
\left|\log p_{\theta}\left(Y_{k} \mid X_{0}=x_{0}, Y_{-s+1}^{k-1}\right)-\log \bar{p}_{\theta}\left(Y_{k} \mid Y_{-s+1}^{k-1}\right)\right| & \leq \frac{\left|p_{\theta}\left(Y_{k} \mid X_{0}=x_{0}, Y_{-s+1}^{k-1}\right)-\bar{p}_{\theta}\left(Y_{k} \mid Y_{-s+1}^{k-1}\right)\right|}{p_{1,-} \int_{E} p_{2, \theta}\left(Y_{k} \mid x_{k}, Y_{k-s}^{k-1}\right) d \mathfrak{m}_{E}\left(x_{k}\right)} \\
& \leq \rho^{k-1} \frac{p_{1,+}}{p_{1,-}}=\frac{\rho^{k-1}}{1-\rho} \overline{\mathbb{P}}_{\theta}-\text { a.s. }
\end{aligned}
$$

and so (A.6) since $\overline{\mathbb{P}}_{\theta^{*}}$ is absolutely continuous with respect to $\overline{\mathbb{P}}_{\theta}$ (for all $\theta$ ).

\section{A.2. Asymptotic behavior of the log-likelihood}

The idea is to approximate $n^{-1} \ell_{n}(\theta)$ by $n^{-1} \sum_{k=1}^{n} \log p_{\theta}\left(Y_{k} \mid Y_{-\infty}^{k-1}\right)$. To this end, we define, for any $k \geq 0$, any $m \geq 0$ and any $x_{0} \in E$, the following quantities

$$
\Delta_{k, m, x}(\theta):=\log \bar{p}_{\theta}\left(Y_{k} \mid Y_{-m-s+1}^{k-1}, X_{-m}=x\right) \text { and } \Delta_{k, m}(\theta):=\log \bar{p}_{\theta}\left(Y_{k} \mid Y_{-m-s+1}^{k-1}\right) .
$$

With these notations, we have

$$
\ell_{n}(\theta)=\sum_{k=1}^{n} \Delta_{k, 0}(\theta) \text { and } \ell_{n}\left(\theta, x_{0}\right)=\sum_{k=1}^{n} \Delta_{k, 0, x_{0}}(\theta) .
$$

Lemma A.4. (as [10], Lem. 3) With the notation $\rho$ introduced in Corollary A.2, we have $\overline{\mathbb{P}}_{\theta^{*}-\text { almost surely }}$

$$
\begin{array}{r}
\forall m, m^{\prime} \geq 0, \sup _{\theta \in \Theta} \sup _{x, x^{\prime} \in E}\left|\Delta_{k, m, x}(\theta)-\Delta_{k, m^{\prime}, x^{\prime}}(\theta)\right| \leq \rho^{k+\min \left(m, m^{\prime}\right)-1} /(1-\rho), \\
\forall m \geq 0, \sup _{\theta \in \Theta x \in E}\left|\Delta_{k, m, x}(\theta)-\Delta_{k, m}(\theta)\right| \leq \rho^{k+m-1} /(1-\rho), \\
\sup _{\theta} \sup _{m \geq 0} \sup _{x \in E}\left|\Delta_{k, m, x}(\theta)\right| \leq \max \left(\left|\log \left(p_{1,+} b_{+}\left(Y_{k-s}^{k}\right)\right)\right|,\left|\log \left(p_{1,-} b_{-}\left(Y_{k-s}^{k}\right)\right)\right|\right),
\end{array}
$$


with

$$
b_{-}\left(y_{k-s}^{k}\right):=\inf _{\theta} \int_{E} p_{2, \theta}\left(y_{k} \mid x, y_{k-s}^{k-1}\right) d \mathfrak{m}_{E}(x),
$$

and

$$
b_{+}\left(y_{k-s}^{k}\right):=\sup _{\theta} \int_{E} p_{2, \theta}\left(y_{k} \mid x, y_{k-s}^{k-1}\right) d \mathfrak{m}_{E}(x) .
$$

Proof. Assume that $m \leq m^{\prime}$. We have

$$
e^{\Delta_{k, m, x}(\theta)}=\int_{E^{2}} q_{\theta}\left(x_{k}, Y_{k} \mid x_{k-1}, Y_{k-s}^{k-1}\right) p_{\theta}\left(X_{k-1}=x_{k-1} \mid X_{-m}=x, Y_{-m-s+1}^{k-1}\right) d \mathfrak{m}_{E}^{\otimes 2}\left(x_{k}, x_{k-1}\right) .
$$

Observe moreover that, due to Fact A.1, we have

$$
e^{\Delta_{k, m^{\prime}, x^{\prime}}(\theta)}=\int_{E} e^{\Delta_{k, m, x^{\prime \prime}}(\theta)} p_{\theta}\left(X_{-m}=x^{\prime \prime} \mid X_{-m^{\prime}}=x^{\prime}, Y_{-m^{\prime}-s+1}^{k-1}\right) d \mathfrak{m}_{E}\left(x^{\prime \prime}\right) .
$$

Therefore, according to Corollary A.2, we obtain

$$
\begin{aligned}
\left|e^{\Delta_{k, m, x}(\theta)}-e^{\Delta_{k, m^{\prime}, x^{\prime}}(\theta)}\right| & \leq \sup _{x^{\prime \prime} \in E}\left|e^{\Delta_{k, m, x}(\theta)}-e^{\Delta_{k, m, x^{\prime \prime}}(\theta)}\right| \\
& \leq p_{1,+} \rho^{k+m-1} \int_{E} p_{2, \theta}\left(Y_{k} \mid x_{k}, Y_{k-s}^{k-1}\right) d \mathfrak{m}_{E}\left(x_{k}\right) .
\end{aligned}
$$

Since

$$
\left|e^{\Delta_{k, m, x}(\theta)}\right| \geq p_{1,-} \int_{E} p_{2, \theta}\left(Y_{k} \mid x_{k}, Y_{k-s}^{k-1}\right) d \mathfrak{m}_{E}\left(x_{k}\right),
$$

we get the first point. The proof of the second point follows exactly the same scheme with the use of the following formula

$$
e^{\Delta_{k, m}(\theta)}=\int_{E} e^{\Delta_{k, m, x_{-m}}(\theta)} \bar{p}_{\theta}\left(X_{-m}=x_{-m} \mid Y_{-m-s+1}^{k-1}\right) d \mathfrak{m}_{E}\left(x_{-m}\right) .
$$

The last point comes from the fact that

$$
p_{1,-} \int_{E} p_{2, \theta}\left(Y_{k} \mid x_{k}, Y_{k-s}^{k-1}\right) d \mathfrak{m}_{E}\left(x_{k}\right) \leq e^{\Delta_{k, m, x}(\theta)} \leq p_{1,+} \int_{E} p_{2, \theta}\left(Y_{k} \mid x_{k}, Y_{k-s}^{k-1}\right) d \mathfrak{m}_{E}\left(x_{k}\right) .
$$

Due to (A.9), we get that, $\overline{\mathbb{P}}_{\theta^{*} \text {-a.s., }}\left(\Delta_{k, m, x}(\theta)\right)_{m}$ is a (uniform in $\left.(k, x, \theta)\right)$ Cauchy sequence and so converges uniformly in $(k, x, \theta)$ to some $\Delta_{k, \infty, x}(\theta)$.

Due to (A.9) and (A.10), $\Delta_{k, \infty, x}(\theta)$ does not depend on $x$ and will be denoted by $\Delta_{k, \infty}(\theta)$. Moreover we have $\Delta_{k, \infty}(\theta)=\Delta_{0, \infty}(\theta) \circ \tau^{k}$.

Due to (A.11), (2.1), (2.2) and (2.3), $\left(\Delta_{k, m, x}(\theta)\right)_{k, m, x}$ is uniformly bounded in $\mathbb{L}^{1}\left(\overline{\mathbb{P}}_{\theta^{*}}\right)$. Therefore $\Delta_{k, \infty}(\theta)$ is in $\mathbb{L}^{1}\left(\overline{\mathbb{P}}_{\theta^{*}}\right)$. Let us write

$$
\ell(\theta):=\overline{\mathbb{E}}_{\theta^{*}}\left[\Delta_{0, \infty}(\theta)\right]
$$

Since $\left(\Omega, \mathcal{F}, \overline{\mathbb{P}}_{\theta^{*}}, \tau\right)$ is ergodic, from the Birkhoff-Khinchine ergodic theorem, we have

$$
\lim _{n \rightarrow+\infty} n^{-1} \sum_{k=1}^{n} \Delta_{k, \infty}(\theta)=\ell(\theta) \quad \overline{\mathbb{P}}_{\theta^{*}}-\text { a.s. and in } \mathbb{L}^{1}\left(\overline{\mathbb{P}}_{\theta^{*}}\right) \text {. }
$$

Now, due to (A.9) and (A.10) applied with $m=0$, we obtain

$$
\sum_{k=1}^{n} \sup _{\theta}\left|\Delta_{k, 0}(\theta)-\Delta_{k, \infty}(\theta)\right| \leq \frac{2}{(1-\rho)^{2}} \quad \overline{\mathbb{P}}_{\theta^{*}}-\text { a.s. }
$$


Now, putting together (A.8), (A.10), (A.12) and (A.13), we have

Corollary A.5.

$$
\lim _{n \rightarrow+\infty} n^{-1} \ell_{n}\left(\theta, x_{0}\right)=\lim _{n \rightarrow+\infty} n^{-1} \ell_{n}(\theta)=\ell(\theta), \quad \overline{\mathbb{P}}_{\theta^{*}}-\text { a.s. }
$$

Still following [10], we have the next lemma insuring the continuity of $\theta \mapsto \ell(\theta)$.

Lemma A.6. (as [10], Lem. 4) For all $\theta \in \Theta$,

$$
\lim _{\delta \rightarrow 0} \overline{\mathbb{E}}_{\theta^{*}}\left[\sup _{\left|\theta-\theta^{\prime}\right| \leq \delta}\left|\Delta_{0, \infty}(\theta)-\Delta_{0, \infty}\left(\theta^{\prime}\right)\right|\right]=0 .
$$

Proof. We recall that $\Delta_{0, \infty}=\lim _{m \rightarrow \infty} \Delta_{0, m, x}(\theta)$ (for every $x \in E$ ) with

$$
\Delta_{0, m, x}(\theta)=\log \frac{\int_{E^{m}} \prod_{\ell=-m+1}^{0} q_{\theta}\left(x_{\ell}, Y_{\ell} \mid x_{\ell-1}, Y_{\ell-s}^{\ell-1}\right) d m_{E}^{\otimes m}\left(x_{-m+1}^{0}\right) d \delta_{x}\left(x_{-m}\right)}{\int_{E^{m-1}} \prod_{\ell=-m+1}^{-1} q_{\theta}\left(x_{\ell}, Y_{\ell} \mid x_{\ell-1}, Y_{\ell-s}^{\ell-1}\right) d m_{E}^{\otimes(m-1)}\left(x_{-m+1}^{-1}\right) d \delta_{x}\left(x_{-m}\right)} .
$$

Since the maps $\theta \mapsto q_{\theta}\left(x_{\ell}, y_{\ell} \mid x_{\ell-1}, y_{\ell-s}^{\ell-1}, y_{\ell}\right)$ are continuous, $\Delta_{0, m, x}$ is $\overline{\mathbb{P}}_{\theta^{*}-\text { almost surely continuous. The uniform }}$

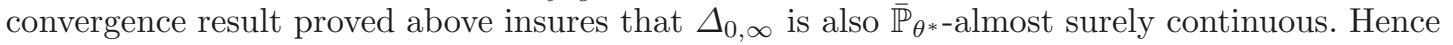

$$
\forall \theta, \quad \lim _{\delta \rightarrow 0} \sup _{\theta^{\prime}:\left|\theta-\theta^{\prime}\right| \leq \delta}\left|\Delta_{0, \infty}(\theta)-\Delta_{0, \infty}\left(\theta^{\prime}\right)\right|=0 \quad \overline{\mathbb{P}}_{\theta^{*}}-\text { a.s. }
$$

Now, the result follows from the Lebesgue dominated convergence theorem, due to (A.11), (2.1), (2.2) and (2.3).

Lemma A.7. (as [10], Prop. 2) We have

$$
\lim _{n \rightarrow+\infty} \sup _{\theta \in \Theta}\left|n^{-1} \ell_{n}\left(\theta, x_{0}\right)-\ell(\theta)\right|=0, \quad \overline{\mathbb{P}}_{\theta^{*}}-\text { a.s. }
$$

Lemma A.7 can be deduced exactly as in the proof of ([10], Prop. 2). We do not rewrite the proof, but mention that it uses (A.6), the compacity of $\Theta$, the continuity of $\ell$, (A.13), the ergodicity of $\overline{\mathbb{P}}_{\theta^{*}}$ and Lemma A.6.

Lemma A.8. (as [10], Lem. 5) For every $k \leq \ell$, we have

$$
\lim _{j \rightarrow-\infty} \sup _{i \leq j}\left|\bar{p}_{\theta}\left(Y_{k}^{\ell} \mid Y_{i-s+1}^{j}\right)-\bar{p}_{\theta}\left(Y_{k}^{\ell}\right)\right|=0 \quad \text { in } \overline{\mathbb{P}}_{\theta^{*}}-\text { probability. }
$$

Proof. Let us write $G\left(y_{-s}^{0}\right):=\int_{E} p_{2, \theta}\left(y_{0} \mid x, y_{-s}^{-1}\right) d \mathfrak{m}_{E}(x)$ and $\tilde{G}\left(y_{0}\right):=\sup _{y_{-s}^{-1}} G\left(y_{-s}^{0}\right)$. As in the proof of ([10], Lem. 5), we observe that, by stationarity, it is enough to prove that

$$
\forall \ell>0, \quad \lim _{k \rightarrow+\infty} \sup _{i \geq 0}\left|\bar{p}_{\theta}\left(Y_{k}^{k+\ell} \mid Y_{-i-s+1}^{0}\right)-\bar{p}_{\theta}\left(Y_{k}^{k+\ell}\right)\right|=0 \text { in } \overline{\mathbb{P}}_{\theta^{*}}-\text { probability }
$$

and we write

$$
\left|\bar{p}_{\theta}\left(Y_{k}^{k+\ell} \mid Y_{-i-s+1}^{0}\right)-\bar{p}_{\theta}\left(Y_{k}^{k+\ell}\right)\right|=\left|\int_{E^{2} \times K^{2 s}} A_{k}\left(B_{k}^{\prime}-B_{k}^{\prime \prime}\right) C_{i} d \mathfrak{m}_{E}^{\otimes 2}\left(x_{s}, x_{k-1}\right) d \mathfrak{m}_{K}^{\otimes 2 s}\left(y_{1}^{s}, y_{k-s}^{k-1}\right)\right|,
$$

with

$$
A_{k}:=p_{\theta}\left(Y_{k}^{k+\ell} \mid X_{k-1}=x_{k-1}, Y_{k-s}^{k-1}=y_{k-s}^{k-1}\right) \leq \tilde{A}_{k}:=p_{1,+}^{\ell+1} \prod_{j=k+s}^{k+\ell} G\left(Y_{j-s}^{j}\right) \prod_{j=k}^{k+s-1} \tilde{G}\left(Y_{j}\right),
$$

(due to (A.4) and to (2.1)) with

$$
B_{k}^{\prime}:=p_{\theta}\left(X_{k-1}=x_{k-1}, Y_{k-s}^{k-1}=y_{k-s}^{k-1} \mid X_{s}=x_{s}, Y_{1}^{s}=y_{1}^{s}\right)=Q_{\theta}^{*(k-s-1)}\left(x_{k-1}, y_{k-s}^{k-1} \mid x_{s}, y_{1}^{s}\right),
$$


with

$$
B_{k}^{\prime \prime}:=\bar{p}_{\theta}\left(X_{k-1}=x_{k-1}, Y_{k-s}^{k-1}=y_{k-s}^{k-1}\right)=h_{\theta}\left(x_{k-1}, y_{k-s}^{k-1}\right)
$$

and with

$$
C_{i}:=\bar{p}_{\theta}\left(X_{s}=x_{s}, Y_{1}^{s}=y_{1}^{s} \mid Y_{-i-s+1}^{0}\right)
$$

Let us write

$$
B_{k}:=\int_{E \times K^{s}}\left|B_{k}^{\prime}-B_{k}^{\prime \prime}\right| d \mu\left(x_{k-1}, y_{k-s}^{k-1}\right)
$$

We have

$$
\left|\bar{p}_{\theta}\left(Y_{k}^{k+\ell} \mid Y_{i-s+1}^{0}\right)-\bar{p}_{\theta}\left(Y_{k}^{k+\ell}\right)\right| \leq \tilde{A}_{k} \int_{E \times K^{s}} B_{k} C_{i} d \mu\left(x_{s}, y_{1}^{s}\right) .
$$

On the one hand, due to $(2.5), B_{k}=B_{k}\left(x_{s}, y_{1}^{s}\right)$ converges to 0 as $k$ goes to infinity, for $\mu$-almost every $\left(x_{s}, y_{1}^{s}\right)$ (and this quantity is bounded by 1 ). On the other hand, on $\left\{Y_{-i-s+1}^{0}=y_{-i-s+1}^{0}\right\}$, we have

$$
\begin{aligned}
C_{i} & =\int_{E^{s}} \prod_{j=1}^{s} q_{\theta}\left(x_{j}, y_{j} \mid x_{j-1}, y_{j-s}^{j-1}\right) \bar{p}_{\theta}\left(X_{0}=x_{0} \mid Y_{-i-s+1}^{0}=y_{-i-s+1}^{0}\right) d \mathfrak{m}_{E}^{\otimes s}\left(x_{0}^{s-1}\right) \\
& \leq p_{1,+} H\left(x_{s}, y_{-s+1}^{s}\right)
\end{aligned}
$$

with

$$
H\left(x_{s}, y_{-s+1}^{s}\right):=\int_{E^{s-1}} \prod_{j=2}^{s} p_{1, \theta}\left(x_{j} \mid x_{j-1}, y_{j-1}\right) \prod_{j=1}^{s} p_{2, \theta}\left(y_{j} \mid x_{j}, y_{j-s}^{j-1}\right) d \mathfrak{m}_{E}^{\otimes s}\left(x_{1}^{s-1}\right),
$$

and

$$
\forall y_{-s+1}^{0}, \quad \int_{E \times K^{s}} H\left(x_{s}, y_{-s+1}^{s}\right) d \mu\left(x_{s}, y_{1}^{s}\right)=1
$$

Therefore, by the Lebesgue dominated convergence theorem, we obtain

$$
\lim _{k \rightarrow+\infty} \sup _{i \leq 0} \int_{E \times K^{s}} B_{k} C_{i} d \mu\left(x_{s}, y_{1}^{s}\right)=0 \quad \overline{\mathbb{P}}_{\theta^{*}}-\text { a.s. }
$$

Of course, this convergence also holds in $\overline{\mathbb{P}}_{\theta^{*}}$-probability. Now, since, for every $k, \tilde{A}_{k}$ is a real valued random variable (see (2.4)) with the same distribution as $p_{1,+}^{\ell+1} \prod_{j=s}^{\ell} G\left(Y_{j-s}^{j}\right) \prod_{j=0}^{s-1} \tilde{G}\left(Y_{j}\right)$, we obtain the result.

Lemma A.9. ([10], Lems. $6 \&$ 7, Prop. 3) For every $\theta \in \Theta, \ell(\theta) \leq \ell\left(\theta^{*}\right)$. Furthermore

$$
\ell(\theta)=\ell\left(\theta^{*}\right) \Rightarrow \overline{\mathbb{P}}_{\theta}^{Y}=\overline{\mathbb{P}}_{\theta^{*}}^{Y} .
$$

Elements of the proof. We do not rewrite the proof of this lemma, the reader can follow the proofs of ([10], Lems. 6 and 7, Prop. 3) (using Lem. A.8 and Kullback-Leibler divergence functions). The only adaptations to make concern the proof of ([10], Lem. 7) which, due to our slightly weaker hypothesis (2.4), are the following facts. Following the proof of Lemma A.8, observe that, due to (2.1), (A.4) and (A.3), on $\left\{Y_{-s+1}^{p}=y_{-s+1}^{p}, Y_{-m-s+1}^{-k}=\right.$ $\left.y_{-m-s+1}^{-k}\right\}, \bar{p}_{\theta}\left(Y_{-s+1}^{p} \mid Y_{-m-s+1}^{-k}\right)$ is between

$$
p_{1,-}^{p+s} \int_{E \times K^{s}} \prod_{j=-s+1}^{p} G\left(y_{j-s}^{j}\right) \bar{p}_{\theta}\left(X_{-s}=x_{-s}, Y_{-2 s+1}^{-s}=y_{-2 s+1}^{-s} \mid Y_{-m-s+1}^{-k}\right) d \mu\left(x_{-s}, y_{-2 s+1}^{-s}\right)
$$

and

$$
p_{1,+}^{p+s} \int_{E \times K^{s}} \prod_{j=-s+1}^{p} G\left(y_{j-s}^{j}\right) \bar{p}_{\theta}\left(X_{-s}=x_{-s}, Y_{-2 s+1}^{-s}=y_{-2 s+1}^{-s} \mid Y_{-m-s+1}^{-k}\right) d \mu\left(x_{-s}, y_{-2 s+1}^{-s}\right),
$$


with $G\left(y_{-s}^{0}\right):=\int_{E} p_{2, \theta}\left(y_{0} \mid x, y_{-s}^{-1}\right) d \mathfrak{m}_{E}(x)$. Therefore we have

$$
\frac{p_{1,-}^{p+s}}{p_{1,+}^{s}} \prod_{j=1}^{p} G\left(Y_{j-s}^{j}\right) \leq \bar{p}_{\theta}\left(Y_{1}^{p} \mid Y_{-s+1}^{0}, Y_{-m-s+1}^{-k}\right)=\frac{\bar{p}_{\theta}\left(Y_{-s+1}^{p} \mid Y_{-m-s+1}^{-k}\right)}{\bar{p}_{\theta}\left(Y_{-s+1}^{0} \mid Y_{-m-s+1}^{-k}\right)} \leq \frac{p_{1,+}^{p+s}}{p_{1,-}^{s}} \prod_{j=1}^{p} G\left(Y_{j-s}^{j}\right) .
$$

Due to $(2.2)$ and (2.3), we obtain

$$
\overline{\mathbb{E}}_{\theta^{*}}\left[\sup _{k} \sup _{m \geq k}\left|\log \left(\bar{p}_{\theta}\left(Y_{1}^{p} \mid Y_{-s+1}^{0}, Y_{-m-s+1}^{-k}\right)\right)\right|\right]<\infty,
$$

which enables the adaptation of the proof of ([10], Lem. 7).

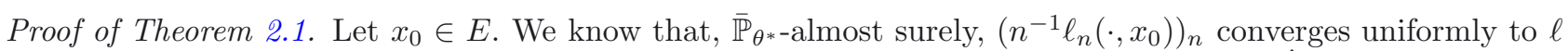
which admits a maximum $\ell\left(\theta^{*}\right)$. Since $\ell_{n}\left(\cdot, x_{0}\right)$ is continuous on $\Theta$ and since $\Theta$ is compact, $\hat{\theta}_{n, x_{0}}$ is well defined. Moreover, the limit values of $\left(\hat{\theta}_{n, x_{0}}\right)_{n}$ are contained in

$$
\left\{\theta \in \Theta: \ell(\theta)=\ell\left(\theta^{*}\right)\right\} \subseteq\left\{\theta \in \Theta: \overline{\mathbb{P}}_{\theta}^{Y}=\overline{\mathbb{P}}_{\theta^{*}}^{Y}\right\}
$$

Assume now that $Q_{\theta^{*}}$ is aperiodic and positive Harris recurrent, following the proof of ([10], Thm. 5), we have $\lim _{n \rightarrow+\infty} \ell\left(\hat{\theta}_{n, x_{0}}\right)=\ell\left(\theta^{*}\right)$ almost surely for any initial measure and we conclude as above.

\section{Appendix B. Identifiability for the Gaussian model: Proof of Proposition 3.6}

Assume that $\overline{\mathbb{P}}_{\theta_{1}}^{Y}=\overline{\mathbb{P}}_{\theta_{2}}^{Y}$. In particular, we have

$$
\bar{p}_{\theta_{1}}\left(Y_{k}=y_{k} \mid Y_{k-s}^{k-1}=y_{k-s}^{k-1}\right)=\bar{p}_{\theta_{2}}\left(Y_{k}=y_{k} \mid Y_{k-s}^{k-1}=y_{k-s}^{k-1}\right), \text { for } \overline{\mathbb{P}}_{\theta_{1}}^{Y_{k-s}^{k}}-\text { a.e. } y_{k-s}^{k},
$$

and thus

$$
\sum_{x=1}^{2} \overline{\mathbb{P}}_{\theta_{1}}\left(X_{k}=x \mid y_{k-s}^{k-1}\right) p_{2, \theta_{1}}\left(y_{k} \mid x, y_{k-s}^{k-1}\right)=\sum_{x=1}^{2} \overline{\mathbb{P}}_{\theta_{2}}\left(X_{k}=x \mid y_{k-s}^{k-1}\right) p_{2, \theta_{2}}\left(y_{k} \mid x, y_{k-s}^{k-1}\right),
$$

for $\overline{\mathbb{P}}_{\theta_{1}}^{Y_{k-s}^{k}}$-almost every $y_{k-s}^{k}$. Since $\bar{p}_{\theta_{1}}\left(y_{k-s}^{k}\right)>0$ (the invariant pdf $h_{1}$ satisfies $h_{1}>0$ and the transition pdf $q_{\theta}$ satisfies $q_{\theta}>0$ by construction), this last equality also holds for Lebesgue almost every $y_{k-s}^{k}$. According to [22], finite mixtures of Gaussian distribution are identifiable. Due to (3.2), this implies in particular that if

$$
\sum_{x=1}^{2} \pi_{x}^{(1)} \mathcal{N}\left(y ; a_{x}^{(1)}, \sigma_{x}^{(1)}\right)=\sum_{x=1}^{M} \pi_{x}^{(2)} \mathcal{N}\left(y ; a_{x}^{(2)}, \sigma_{x}^{(2)}\right) \text { for }- \text { a.e. } y,
$$

with $\left(a_{1}^{(1)}, \sigma_{1}^{(1)}\right) \neq\left(a_{2}^{(1)}, \sigma_{2}^{(1)}\right), \pi_{1}^{(1)}>0$ and $\pi_{2}^{(1)}>0$, then there exists a permutation $\tau:\{1,2\} \rightarrow\{1,2\}$ such that $\left(a_{x}^{(1)}, \sigma_{x}^{(1)}\right)=\left(a_{\tau(x)}^{(2)}, \sigma_{\tau(x)}^{(2)}\right)$ and $\pi_{1}^{(x)}=\pi_{2}^{(\tau(x))}$. Therefore, since for every $x \in\{1,2\}$ and for Lebesgue almost every $y_{k-s}^{k-1}, \overline{\mathbb{P}}_{\theta_{1}}\left(X_{k}=x \mid y_{k-s}^{k-1}\right)>0$ (since $h_{\theta_{1}}>0$ ), for Lebesgue almost every $y_{k-s}^{k-1}$ there exists a permutation $\tau_{y}=\tau_{y_{k-s}^{k-1}}$ of $\{1, \ldots, M\}$ such that,

$$
\forall x \in\{1,2\}, \quad\left(\beta_{0,(1)}^{(x)}+\sum_{\ell=1}^{s} \beta_{\ell,(1)}^{(x)} y_{k-\ell}, \sigma_{(1)}^{(x)}\right)=\left(\beta_{0,(2)}^{\left(\tau_{y}(x)\right)}+\sum_{\ell=1}^{s} \beta_{\ell,(2)}^{\left(\tau_{y}(x)\right)} y_{k-\ell}, \sigma_{(2)}^{\left(\tau_{y}(x)\right)}\right) .
$$

Recall that we have assumed (for the first model)

$$
\left(\beta_{0,(1)}^{(1)}, \beta_{1,(1)}^{(1)}, \ldots, \beta_{s,(1)}^{(1)}, \sigma_{(1)}^{(1)}\right) \neq\left(\beta_{0,(1)}^{(2)}, \beta_{1,(1)}^{(2)}, \ldots, \beta_{s,(1)}^{(2)}, \sigma_{(1)}^{(2)}\right)
$$


which implies

$$
\left(\beta_{0,(1)}^{(1)}+\sum_{\ell=1}^{s} \beta_{\ell,(1)}^{(1)} y_{k-\ell}, \sigma_{(1)}^{(1)}\right) \neq\left(\beta_{0,(1)}^{(2)}+\sum_{\ell=1}^{s} \beta_{\ell,(1)}^{(2)} y_{k-\ell}, \sigma_{(1)}^{(2)}\right),
$$

for Lebesgue almost every $y_{k-s}^{k-1}$. Since the set of permutations of $\{1, \ldots, M\}$ is finite, there exists a positive Lebesgue measure subset of $\mathbb{T}^{s}$ on which the permutation is the same permutation $\tau$. From this, we deduce that, for all $x \in\{1,2\}$ and $y \in \mathbb{R}$,

$$
\left(\beta_{0,(1)}^{(x)}, \beta_{1,(1)}^{(x)}, \ldots, \beta_{r,(1)}^{(x)}, \sigma_{(1)}^{(x)}\right)=\left(\beta_{0,(2)}^{(\tau(x))}, \beta_{1,(2)}^{(\tau(x))}, \ldots, \beta_{r,(2)}^{(\tau(x))}, \sigma_{(2)}^{(\tau(x))}\right)
$$

and

$$
\begin{aligned}
p_{1, \theta_{1}}(x \mid x, y) & =\pi_{-,(1)}^{(x)}+\frac{1-\pi_{-,(1)}^{(x)}-\pi_{+,(1)}^{(x)}}{1+\exp \left(\lambda_{0,(1)}^{(x)}+\lambda_{1,(1)}^{(x)} y\right)} \\
& =\pi_{-,(2)}^{(\tau(x))}+\frac{1-\pi_{-,(2)}^{(\tau(x))}-\pi_{+,(2)}^{(\tau(x))}}{1+\exp \left(\lambda_{0,(2)}^{(\tau(x))}+\lambda_{1,(2)}^{(\tau(x))} y\right)}=p_{1, \theta_{2}}(\tau(x) \mid \tau(x), y) .
\end{aligned}
$$

If $\theta_{1}$ and $\theta_{2}$ are in $\Theta^{\prime}$ then $\lambda_{1,(i)}^{(x)} \neq 0$ for $i \in\{1,2\}$ and looking at the asymptotic behavior of the terms which appear in (B.1) when $y \rightarrow \pm \infty$ permits to show that $\pi_{-,(1)}^{(x)}=\pi_{-,(2)}^{(x)}, \pi_{+,(1)}^{(x)}=\pi_{+,(2)}^{(x)}$. We can then easily deduce that $\lambda_{0,(1)}^{(x)}=\lambda_{0,(2)}^{(x)}$ and $\lambda_{1,(1)}^{(x)}=\lambda_{1,(2)}^{(x)}$ and thus that $\theta_{1}=\theta_{2}$.

If $\theta_{1}$ and $\theta_{2}$ are in $\Theta^{\prime \prime}$, then we directly obtain that $\pi_{-,(1)}^{(x)}=\pi_{-,(2)}^{(x)}=\pi_{+,(1)}^{(x)}=\pi_{-,(1)}^{(x)}=\pi_{0}$ and then that $\theta_{1}=\theta_{2}$.

\section{Appendix C. Identifiability for the Rainfall model: Proof of Proposition 4.4}

Assume that $\overline{\mathbb{P}}_{\theta_{1}}^{Y}=\overline{\mathbb{P}}_{\theta_{2}}^{Y}$. First, we use the fact that

$$
\bar{p}_{\theta_{1}}\left(Y_{k}=y_{k} \mid Y_{k-s}^{k-1}=y_{k-s}^{k-1}\right)=\bar{p}_{\theta_{2}}\left(Y_{k}=y_{k} \mid Y_{k-s}^{k-1}=y_{k-s}^{k-1}\right) \text { for } \overline{\mathbb{P}}_{\theta_{1}}^{Y_{k-s}^{k}}-\text { a.e. } y_{k-s}^{k},
$$

to prove that

$$
\left(\pi_{i,(1)}^{(x)}, \alpha_{i,(1)}^{(x)}, \beta_{i,(1)}^{(x)}\right)_{i, x}=\left(\pi_{i,(2)}^{(x)}, \alpha_{i,(2)}^{(x)}, \beta_{i,(2)}^{(x)}\right)_{i, x} .
$$

Using (C.1) on the set $\left\{r_{k}^{(i)}>0, \forall i \in\{1, \ldots, \ell\}\right\}$, we conclude that there exists a permutation $\tau$ of $\{1, \ldots, M\}$ such that, for every $i \in\{1, \ldots, \ell\}$ and every $x \in\{1, \ldots, M\}$, we have

$$
\left(\alpha_{i,(1)}^{(x)}, \beta_{i,(1)}^{(x)}\right)=\left(\alpha_{i,(2)}^{(\tau(x))}, \beta_{i,(2)}^{(\tau(x))}\right)
$$

and

$$
\overline{\mathbb{P}}_{\theta_{1}}\left(X_{k}=x \mid Y_{k-s}^{k-1}=y_{k-s}^{k-1}\right) \prod_{i=1}^{\ell} \pi_{i,(1)}^{(x)}=\overline{\mathbb{P}}_{\theta_{2}}\left(X_{k}=x \mid Y_{k-s}^{k-1}=y_{k-s}^{k-1}\right) \prod_{i=1}^{\ell} \pi_{i,(2)}^{(\tau(x))} .
$$

Now, for every $J \subseteq\{1, \ldots, \ell\}$, we use (C.1) on the set $\left\{r_{k}^{(j)}>0, \forall j \in J, r_{k}^{(i)}=0, \forall i \notin J\right\}$. Due to (C.2) and since $\theta_{1}$ satisfies (4.8), we obtain

$$
\overline{\mathbb{P}}_{\theta_{1}}\left(X_{k}=x \mid Y_{k-s}^{k-1}=y_{k-s}^{k-1}\right) \prod_{j \in J} \pi_{j,(1)}^{(x)} \prod_{i \notin J}\left(1-\pi_{i,(1)}^{(x)}\right)=\overline{\mathbb{P}}_{\theta_{2}}\left(X_{k}=x \mid Y_{k-s}^{k-1}=y_{k-s}^{k-1}\right) \prod_{j \in J} \pi_{j,(2)}^{(\tau(x))} \prod_{i \notin J}\left(1-\pi_{i,(2)}^{(\tau(x))}\right) .
$$


From which, we conclude

$$
\forall i \in\{1, \ldots, \ell\}, \forall x \in\{1, \ldots, M\}, \quad \pi_{i,(1)}^{(x)}=\pi_{i,(2)}^{(\tau(x))} .
$$

Now it remains to prove that $\left(q_{x, x^{\prime},(1)}, \mu_{x, x^{\prime},(1)}\right)=\left(q_{\tau(x), \tau\left(x^{\prime}\right),(2)}, \mu_{\tau(x), \tau\left(x^{\prime}\right),(2)}\right)$. To this hand, as for the AR model (see Appendix B), we use the fact that

$$
\bar{p}_{\theta_{1}}\left(Y_{k}=y_{k}, Y_{k+1}=y_{k+1} \mid Y_{k-s}^{k-1}=y_{k-s}^{k-1}\right)=\bar{p}_{\theta_{2}}\left(Y_{k}=y_{k}, Y_{k+1}=y_{k+1} \mid Y_{k-s}^{k-1}=y_{k-s}^{k-1}\right) \text { for } \overline{\mathbb{P}}_{\theta_{1}}^{Y_{k-s}^{k}}-\text { a.e. } y_{k-s}^{k}
$$

and obtain that

$$
\forall x, x^{\prime}, \quad p_{1, \theta_{1}}\left(x^{\prime} \mid x, y_{k}\right)=p_{1, \theta_{2}}\left(\tau\left(x^{\prime}\right) \mid \tau(x), y_{k}\right) \text { for a.e. } y_{k} \text {. }
$$

This implies that

$$
\frac{\tilde{q}_{x, x^{\prime},(1)} \exp \left(-z_{k-1}^{\prime} \tilde{\mu}_{x, x^{\prime},(1)}\right)}{\sum_{x,}, \tilde{q}_{x, x^{\prime \prime},(1)} \exp \left(-z_{k-1}^{\prime} \tilde{\mu}_{x, x^{\prime},(1)}\right)}=\frac{\tilde{q}_{\tau(x), \tau\left(x^{\prime}\right),(2)} \exp \left(-z_{k-1}^{\prime} \tilde{\mu}_{\tau(x), \tau\left(x^{\prime}\right),(2)}\right)}{\sum_{x,} \tilde{q}_{x, \tau\left(x^{\prime \prime}\right),(2)} \exp \left(-z_{k-1}^{\prime} \tilde{\mu}_{x, \tau(x),(2)}\right)},
$$

with $\tilde{q}_{x, x^{\prime},(j)}:=q_{x, x^{\prime},(j)} \exp \left(-\frac{1}{2}\left(\mu_{x, x^{\prime},(j)}\right)^{\prime} \Sigma^{-1} \mu_{x, x^{\prime},(j)}\right)$ and $\tilde{\mu}_{x, x^{\prime},(j)}:=\Sigma^{-1} \mu_{x, x^{\prime},(j)}$. From (C.5), we obtain that

$$
\frac{\tilde{q}_{x, x^{\prime},(1)} \exp \left(-z_{k-1}^{\prime} \tilde{\mu}_{x, x^{\prime},(1)}\right)}{\tilde{q}_{x, x,(1)} \exp \left(-z_{k-1}^{\prime} \tilde{\mu}_{x, x,(1)}\right)}=\frac{\tilde{q}_{\tau(x), \tau\left(x^{\prime}\right),(2)} \exp \left(-z_{k-1}^{\prime} \tilde{\mu}_{\tau(x), \tau\left(x^{\prime}\right),(2)}\right)}{\tilde{q}_{\tau(x), \tau(x),(2)} \exp \left(-z_{k-1}^{\prime} \tilde{\mu}_{\tau(x), \tau(x),(2)}\right)},
$$

and so that, for every $x, x^{\prime} \in\{1, \ldots, M\}$,

$$
\tilde{\mu}_{x, x^{\prime},(1)}-\tilde{\mu}_{x, x,(1)}=\tilde{\mu}_{\tau(x), \tau\left(x^{\prime}\right),(2)}-\tilde{\mu}_{\tau(x), \tau(x),(2)},
$$

and

$$
\frac{\tilde{q}_{x, x^{\prime},(1)}}{\tilde{q}_{x, x,(1)}}=\frac{\tilde{q}_{\tau(x), \tau\left(x^{\prime}\right),(2)}}{\tilde{q}_{\tau(x), \tau(x),(2)}}
$$

Finally, it comes from (C.6) that $\tilde{\mu}_{x, x^{\prime},(1)}=\tilde{\mu}_{\tau(x), \tau\left(x^{\prime}\right),(2)}\left(\right.$ using $\left.\sum_{x "} \tilde{\mu}_{x, x^{\prime \prime},(j)}=0\right)$ and so $\mu_{x, x^{\prime},(1)}=$ $\mu_{\tau(x), \tau\left(x^{\prime}\right),(2)}$. So (C.7) becomes

$$
\frac{q_{x, x^{\prime},(1)}}{q_{x, x,(1)}}=\frac{q_{\tau(x), \tau\left(x^{\prime}\right),(2)}}{q_{\tau(x), \tau(x),(2)}}
$$

which implies that $q_{x, x^{\prime},(1)}=q_{\tau(x), \tau\left(x^{\prime}\right),(2)}\left(\right.$ due to $\sum_{x "} q_{x, x^{\prime \prime},(j)}=1$ ).

\section{Appendix D. Proof of Lemma 4.1}

Let $f$ be any probability pdf wrt $\mu=\mathfrak{m}_{E} \times \mathfrak{m}_{K}$. We have

$$
\begin{aligned}
{\left[Q_{\theta}^{* n}\left(f-h_{\theta}\right)\right]\left(x_{0}, y_{0}\right) } & =\int_{(E \times K)^{n}} \prod_{i=-n+1}^{0} q_{\theta}\left(x_{i}, y_{i} \mid x_{i-1}\right)\left(f-h_{\theta}\right)\left(x_{-n}, y_{-n}\right) d \mathfrak{m}_{E}^{\otimes n}\left(x_{-n}^{-1}\right) d \mathfrak{m}_{K}^{\otimes n}\left(y_{-n}^{-1}\right) \\
& =\int_{E^{n} \times K^{n-1}} \prod_{i=-n+1}^{0} q_{\theta}\left(x_{i}, y_{i} \mid x_{i-1}\right)\left(F-h_{1, \theta}\right)\left(x_{-n}\right) d \mathfrak{m}_{E}^{\otimes n}\left(x_{-n}^{-1}\right) d \mathfrak{m}_{K}^{\otimes(n-1)}\left(y_{-n+1}^{-1}\right),
\end{aligned}
$$

with $F\left(x_{-n}\right):=\int_{K} f\left(x_{-n}, y_{-n}\right) d \mathfrak{m}_{K}\left(y_{-n}\right)$. Now, since $q_{\theta}\left(x_{i}, y_{i} \mid x_{i-1}\right)=p_{1, \theta}\left(x_{i} \mid x_{i-1}\right) p_{2, \theta}\left(y_{i} \mid x_{i}\right)$, we obtain that

$$
\left[Q_{\theta}^{* n}\left(f-h_{1, \theta}\right)\right]\left(x_{0}, y_{0}\right)=p_{2, \theta}\left(y_{0} \mid x_{0}\right) \int_{E^{n}} \prod_{i=-n+1}^{0} p_{1, \theta}\left(x_{i} \mid x_{i-1}\right)\left(F-h_{1, \theta}\right)\left(x_{-n}\right) d \mathfrak{m}_{E}^{\otimes n}\left(x_{-n}^{-1}\right)
$$


Therefore

$$
\left\|Q_{\theta}^{* n}\left(f-h_{\theta}\right)\right\|_{L^{1}\left(\mathfrak{m}_{E} \times \mathfrak{m}_{K}\right)}=\left\|Q_{1, \theta}^{* n}\left(F-h_{1, \theta}\right)\right\|_{L^{1}\left(\mathfrak{m}_{E}\right)} .
$$

Now, let us assume that $p_{2, \theta}>0$ and that $\left(X_{k}\right)_{k}$ is an aperiodic positive Harris recurrent Markov chain. We will use the notations of [21].

Since $\left(X_{k}\right)_{k}$ is positive, it is $\psi$-irreducible (with $\psi=\psi_{0}$ ). Due to the hypothesis on $p_{2, \theta}$, this implies the $\psi$-irreducibility of $\left(X_{k}, Y_{k}\right)_{k}$ (with $\left.\psi=\psi_{0} \times \mathfrak{m}_{K}\right)$.

Moreover $\left(X_{k}, Y_{k}\right)_{k}$ is positive since it admits an invariant probability measure (due to the first point of this result).

The fact that $\left(X_{k}\right)_{k}$ is aperiodic means that, for every $\nu_{M}$-small set $C$ such that $\nu_{M}(C)>0$ for $\left(X_{k}\right)_{k}$, the greatest common divisor of the set $E_{C}$ defined as follows is equal to 1 :

$$
E_{C}:=\left\{n \geq 1: C \text { is } \nu_{n}-\text { small with } \nu_{n}=\delta_{n} \nu_{M} \text { and } \delta_{n}>0\right\} .
$$

Now, let $C^{\prime}$ be a $\nu_{M}^{\prime}$-small set for $\left(X_{k}, Y_{k}\right)_{k}$ with $\nu_{M}^{\prime}\left(C^{\prime}\right)>0$, then for every $\left(x_{0}, y_{0}\right) \in C^{\prime}$ and every $(B, D) \in$ $\mathcal{B}(E) \times \mathcal{B}(K)$, we have $Q_{\theta}^{M} \mathbb{1}_{B \times D}\left(x_{0}, y_{0}\right) \geq \nu_{M}^{\prime}(B \times D)$. Moreover $Q_{\theta}^{M} \mathbb{1}_{B \times D}\left(x_{0}, y_{0}\right)$ is equal to

$$
\int_{E^{M-1}}\left(\int_{B} \prod_{i=1}^{M} p_{1, \theta}\left(x_{i} \mid x_{i-1}\right)\left(\int_{D} p_{2, \theta}\left(y_{M} \mid x_{M}\right) d \mathfrak{m}_{K}\left(y_{M}\right)\right) d \mathfrak{m}_{E}\left(x_{M}\right)\right) d \mathfrak{m}_{E}^{\otimes(M-1)}\left(x_{1}^{M-1}\right) .
$$

Since $Q_{\theta}^{M} \mathbb{1}_{B \times D}\left(x_{0}, y_{0}\right)$ does not depend on $y_{0}$, we obtain

$$
\forall\left(x_{0}, y_{0}\right) \in E \times K, \forall B \in \mathcal{B}(E), \quad Q_{1, \theta}^{M} \mathbb{1}_{B}\left(x_{0}\right)=Q_{\theta}^{M} \mathbb{1}_{B \times K}\left(x_{0}, y_{0}\right) \geq \nu_{M}^{\prime}(B \times K),
$$

and so $C:=\left\{x \in E: \exists y \in K,(x, y) \in C^{\prime}\right\}$ is $\nu_{M}$-small with $\nu_{M}(B)=\nu_{M}^{\prime}(B \times K)$ and $\nu_{M}(C) \geq \nu_{M}^{\prime}\left(C^{\prime}\right)>0$. Moreover $E_{C}=E_{C^{\prime}}$. Indeed, if $C^{\prime}$ is $\nu_{n}^{\prime}$-small with $\nu_{n}^{\prime}=\delta_{n}^{\prime} \nu_{M}^{\prime}$, then $C$ is $\nu_{n}$-small with $\nu_{n}(B)=\nu_{n}^{\prime}(B \times K)=$ $\delta_{n} \nu_{M}(B)$ with $\delta_{n}(x)=\int_{K} \delta_{n}^{\prime}(x, y) d \mathfrak{m}_{K}(y)$; and conversely, if $C$ is $\nu_{n}$-small with $\nu_{n}=\delta_{n} \nu_{M}$, then $C^{\prime}$ is $\nu_{n}^{\prime}$-small with $\nu_{n}^{\prime}(B \times D)=\delta_{n}^{\prime} \nu_{M}^{\prime}(B \times D)$ and with $\delta_{n}^{\prime}(x, y)=\delta_{n}(x) p_{2, \theta}(y \mid x)$. Therefore $\left(X_{k}, Y_{k}\right)_{k}$ is also aperiodic.

Finally, the Harris recurrence property of $\left(X_{k}, Y_{k}\right)_{k}$ follows from the Harris-recurrence of $\left(X_{k}\right)_{k}$ and from $p_{2, \theta}>0$.

\section{REFERENCES}

[1] P. Ailliot, Some theoretical results on Markov-switching autoregressive models with gamma innovations. C. R. Acad. Sci. Ser. I 343 (2006) 271-274.

[2] P. Ailliot and V. Monbet, Markov-switching autoregressive models for wind time series. Environ. Model. Softw. 30 (2012) 92-101.

[3] P. Ailliot, J. Bessac, V. Monbet, F. Pène, Non-homogeneous hidden Markov-switching models for wind time series. J. Stat. Plann. Inference 160 (2015) 75-88.

[4] H.Z. An and F.C. Huang, The geometrical ergodicity of nonlinear autoregressive models. Stat. Sin. 6 (1996) 943-956.

[5] E. Bellone, J.P. Hughes and P. Guttorp, A hidden Markov model for downscaling synoptic atmospheric patterns to precipitation amounts. Climate Res. 15 (2000) 1-12.

[6] O. Cappé, E. Moulines and T. Rydén, Inference in Hidden Markov Models. Springer-Verlag, New York (2005).

[7] R. Chen and R.S. Tsay, On the ergodicity of tar(1) processes. Ann. Appl. Probab. 1 (1991) 613-634.

[8] F. Diebold, J-H Lee and G. Weinbach, Regime Switching with Time-Varying Transition Probabilities. Oxford University Press, Oxford (1994).

[9] R. Douc and C. Matias, Asymptotics of the maximum-likelihood estimator for general hidden Markov models. Bernoulli 7 (2001) 381-420.

[10] R. Douc, E. Moulines and T. Rydn, Asymptotic properties of the maximum likelihood estimator in autoregressive models with Markov regime. Ann. Stat. 32 (2004) 2254-2304.

[11] J. Fan and Q. Yao, Nonlinear Time Series: Nonparametric and Parametric Methods. Springer, New York (2003).

[12] C. Francq and M. Roussignol, Ergodicity of autoregressive processes with Markov-switching and consistency of the maximumlikelihood estimator. Statistics 32 (1998) 151-173.

[13] J.D. Hamilton, A new approach to the economic analysis of nonstationary time series and the business cycle. Econometrica 57 (1989) 357-384. 
[14] J.P Hughes, P. Guttorp and S.P. Charles, A non-homogeneous hidden Markov model for precipitation occurrence. J. R. Stat. Soc. Series C 48 (1999) 15-30.

[15] C. Kim, J. Piger and R. Startz, Estimation of Markov regime-switching regression models with endogenous switching. J. Econ. 143 (2008) 263-273.

[16] V. Krishnamurthy and T. Ryden, Consistent estimation of linear and non-linear autoregressive models with Markov regime. J. Time Ser. Anal. 19 (1998) 291-307.

[17] H.M. Krolzig, Markov-switching vector autoregressions: modelling, statistical inference, and application to business cycle analysis. Lect. Notes Econ. Math. Systems 454 (1997).

[18] F. Le Gland and L. Mevel, Exponential forgetting and geometric ergodicity in hidden Markov models. Math. Control, Signals Syst. 13 (2000) 63-93.

[19] B. Leroux, Maximum-likelihood estimation for hidden Markov models. Stoch. Proc. Appl. 40 (1992) $127-143$.

[20] T. Lindvall, Lectures on the Coupling Method. Corrected reprint of the 1992 original. Dover Publications, Inc., Mineola, NY (2002).

[21] S.P. Meyn, R.L Tweedie and P.W. Glynn, Markov Chains and Stochastic Stability. Cambridge, Cambridge University Press (2009), vol. 2 .

[22] H. Teicher, Identifiability of finite mixtures. Ann. Math. Stat. 34 (1963) 1265-1269.

[23] H. Tong, Non-Linear Time Series: A Dynamical System Approach. Oxford, UK, Oxford University Press (1990).

[24] I. Visser, M.E.J. Raijmakers and P. Molenaar, Confidence intervals for hidden Markov model parameters. British J. Math. Stat. Psychol. 53 (2000) 317-327.

[25] M. Vrac and P. Naveau, Stochastic downscaling of precipitation: From dry events to heavy rainfalls. Water Res. Research 43 (2007) W07402.

[26] J.F. Yao, On square-integrability of an AR process with Markov switching. Stat. Probab. Lett. 52 (2001) $265-270$.

[27] J.F. Yao and J.G. Attali, On stability of nonlinear AR processes with Markov switching. Adv. Appl. Probab. 32 (2000) $394-407$. 